\title{
A dichotomy in the orientation of dust and radio jets in nearby low-power radio galaxies ${ }^{\star}$
}

\author{
G. A. Verdoes Kleijn ${ }^{1}$ and P. T. de Zeeuw ${ }^{2}$ \\ ${ }^{1}$ European Southern Observatory, Karl-Schwarzschild-Strasse 2, 85748, Garching bei München, Germany \\ e-mail: gverdoes@eso.org \\ 2 Sterrewacht Leiden, Postbus 9513, 2300 RA Leiden, The Netherlands \\ e-mail: dezeeuw@strw.leidenuniv.nl
}

Received 28 October 2004 / Accepted 28 January 2005

\begin{abstract}
We examine the properties of central dust in nearby quiescent and active early-type galaxies. The active galaxies are low-power radio galaxies with Fanaroff \& Riley type I or I/II radio jets. We focus on (a) the comparison of the dust distributions in the active and quiescent galaxy samples; and (b) the relation between the radio jet and dust orientations. Our main observational conclusions are: (i) in line with previous studies, the dust detection rate is higher in radio-jet galaxies than in non radio-jet galaxies; (ii) radio galaxies contain a higher fraction of regular dust "ellipses" compared to quiescent galaxies which contain more often irregular dust distributions; (iii) the morphology, size and orientation of dust ellipses and lanes in quiescent early-types and active early-types with kpc-scale radio jets is very similar; (iv) dust ellipses are aligned with the major axis of the galaxy, dust lanes do not show a preferred alignment except for large $(>\mathrm{kpc})$ dust lanes which are aligned with the minor axis of the galaxy; and (v) as projected on the sky, jets do not show a preferred orientation relative to the galaxy major axis (and hence dust ellipses), but jets are preferentially perpendicular to dust lanes.

We show that the dust ellipses are consistent with being nearly circular thin disks viewed at random viewing angles. The lanes are likely warped dust structures, which may be in the process of settling down to become regular disks or are being perturbed by a non-gravitational force. We use the observed dust-jet orientations to constrain the three-dimensional angle $\theta_{\mathrm{DJ}}$ between jet and dust. For dust-lane galaxies, the jet is approximately perpendicular to the dust structure, while for dust-ellipse galaxies there is a much wider distribution of $\theta_{\mathrm{DJ}}$.

We discuss two scenarios that could explain the dust/jet/galaxy orientation dichotomy. If lanes are indeed settling, then the jet orientation apparently is roughly aligned with the angular momentum of the dust before it settles. If lanes are perturbed by a jet-related force, it appears that it causes the dust to move out of its equilibrium plane in the galaxy into a plane which is perpendicular to the jet.
\end{abstract}

Key words. galaxies: active - galaxies: elliptical and lenticular, cD - galaxies: nuclei - galaxies: jets - ISM: dust, extinction

\section{Introduction}

Many early-type galaxies harbour dust and warm (i.e., $T \sim$ $10^{4-5} \mathrm{~K}$ ) gas in their central regions (e.g., Sadler \& Gerhard 1985; Goudfrooij et al. 1994; van Dokkum \& Franx 1995). Evidence is mounting that all early-type galaxies harbour a central supermassive black hole (BH) (e.g., Kormendy \& Gebhardt 2001, and references therein). The dust and gas form potential fuel for the $\mathrm{BH}$ to power an active galactic nucleus (AGN). In the nearby Universe, many, perhaps the majority, of nearby early-type galaxies display some sort of nuclear activity, most often as a modest AGN (e.g., Ho et al. 1997; Kauffmann et al. 2003). Comparing the demography of the fuel reservoirs in

* Based on observations with the NASA/ESA Hubble Space Telescope obtained at the Space Telescope Science Institute, which is operated by the Association of Universities for Research in Astronomy, Incorporated, under NASA contract NAS5-26555. nearby active and quiescent galaxies can shed light on the triggering and/or feeding mechanism of AGN. In a small fraction of the active galaxies the AGN is accompanied by kpc-scale radio jets (e.g., Condon \& Broderick 1988). Here we focus on those radio galaxies. The orientation and kinematics of the dust and gas form a tracer of the stellar potential in addition to the stellar photometry and kinematics (e.g., Merritt \& de Zeeuw 1983). This requires that the interstellar material has settled in the gravitational potential and is unperturbed by collisional forces. The observed distribution of relative orientations of the gas and dust, the jet, and the stellar potential, can constrain the physical processes that govern the orientation of dust and jet and perhaps the jet formation mechanism in radio galaxies.

Many ground-based studies have surveyed the global, kpc-scale, dust content of nearby (distance $<100 \mathrm{Mpc}$ ) quiescent and/or active early-type galaxies (e.g., Sadler \& Gerhard 1985; Veron-Cetty \& Veron 1988; Knapp et al. 1989; 
Roberts et al. 1991; Goudfrooij et al. 1994). With the Hubble Space Telescope (HST) it has become possible to detect routinely central dust distributions, also those with sizes below $1 \mathrm{kpc}$, in nearby galaxies. Using HST/WFPC2 optical broadband photometry, dust is detected in $\sim 50 \%$ of the nearby earlytype galaxies (van Dokkum \& Franx 1995; Tomita et al. 2000; Tran et al. 2001). This detection rate increases to $\sim 90 \%$ for nearby early-type galaxies with radio jets (van Dokkum \& Franx 1995; Verdoes Kleijn et al. 1999). The increased detection rate in radio galaxies suggests a causal connection between extended central ISM distributions and the on-set of nuclear activity and radio jet formation.

Early studies, using ground-based imaging, showed that jets in radio galaxies are roughly perpendicular in the plane of the sky to kpc-scale dust structures (e.g., Kotanyi \& Ekers 1979; Möllenhoff et al. 1992). No relation was found between galaxy and jet orientation (e.g., Battistini et al. 1980; Birkinshaw \& Davies 1985; Sansom et al. 1987) apart from a tendency to avoid large $\gtrsim 70^{\circ}$ angles between galaxy minor axis and radio jet. In studies of the sub kpc-scale dust, based on HST imaging, the perpendicularity of jet and dust in the plane of the sky was also found for radio galaxies (e.g., van Dokkum \& Franx 1995; de Koff et al. 2000; de Ruiter et al. 2002). Furthermore, evidence was reported that also the intrinsic, i.e., three-dimensional, orientation of radio jets is roughly perpendicular to the dust, using samples of radio galaxies which contain dust disks (Capetti \& Celotti 1999; Sparks et al. 2000). In contrast, Schmitt et al. (2002) found in a sample of 20 radio galaxies with regular dust disks that the jets are not roughly perpendicular to the disks in three-dimensional space.

We have studied a sample of nearby Fanaroff \& Riley (1974) type I radio galaxies, the "UGC FR-I sample" (Xu et al. 2000; Verdoes Kleijn et al. 1999, 2002; Noel-Storr et al. 2003). Verdoes Kleijn et al. (1999) describe a dichotomy in apparent dust morphology of the UGC FR-I sample galaxies, which either contain regular dust structures with an elliptical appearance ("ellipses"), or filamentary structures ("lanes"). Ellipses turn out to be aligned with the galaxy major axis while the lanes show no relation to galaxy orientation. Furthermore, most lanes (but only some ellipses) are perpendicular to jets in the skyplane. Clearly, the apparently conflicting results on the dustjet orientation mentioned above might be connected to this relation between dust orientation and morphology. We explore this relation further by inferring the intrinsic three-dimensional relative orientations of jet, dust and stellar content of the host galaxy. With the results of this analysis we hope to constrain the causal connections which lead to the observed orientations between the following three actors: (i) the gravitational potential; (ii) the central ISM; and (iii) the radio jet. We focus on the following questions. Are the properties of the fuel reservoirs different in radio galaxies and non-radio galaxies? Is the dust orientation governed by the gravitational potential? Do jet/AGN related forces affect the dust orientation?

This paper is organized as follows. In Sect. 2 we describe the selection and properties of the radio and non-radio galaxy samples. In Sects. 3 and 4 we compare the dust properties in radio and non-radio galaxies as derived from HST/WFPC2 imaging. In Sect. 5 we describe the relative orientations of jets, dust and the galaxy in the plane of the sky. In Sects. 6 and 7 we determine the constraints placed by the projected orientations on the intrinsic relative orientations in three dimensions for galaxies with dust classified as ellipses. We consider the dust lane galaxies in Sect. 8. In Sect. 9 we discuss the possible origins for the dichotomy in intrinsic orientation of dust and jets found in Sects. 7 and 8. We summarize our results in Sect. 10.

Throughout the paper we use a Hubble constant $H_{0}=$ $75 \mathrm{~km} \mathrm{~s}^{-1} \mathrm{Mpc}^{-1}$.

\section{Samples and data}

We use a complete sample of 21 radio (i.e., "active") galaxies, the UGC FR-I sample, and a sample of 52 non-radio (i.e., "quiescent") galaxies, the UGC non-FR-I sample, to statistically compare dust distributions. We also consider a larger "FR sample", which contains 47 FR-I and FR-I/II radio galaxies in total, to improve the constraints on the three-dimensional relative orientation of jets, dust and stellar hosts. We rely mainly on HST/WFPC2 imaging. The exceptions are 3C 402N, for which we use HST/FOC imaging, and NGC 5128 for which we employ VLT/VIMOS imaging which provides a full view of the kpc-scale dust distribution in this nearby galaxy.

\subsection{The radio galaxy samples}

The UGC FR-I sample is a complete sample of nearby radio galaxies from the UGC catalogue (Nilson et al. 1973). It contains all 21 nearby $\left(v<7000 \mathrm{~km} \mathrm{~s}^{-1}\right)$, elliptical or S0 galaxies in the declination range $-5^{\circ}<\delta<70^{\circ}$ in the UGC catalogue (magnitude $m_{B}<14^{\mathrm{m}} 6$ and angular size $\theta_{\mathrm{p}}>1.0$ ) that are extended radio-loud sources, defined as larger than $10^{\prime \prime}$ at $3 \sigma$ on VLA A-Array maps and brighter than $150 \mathrm{mJy}$ from single-dish flux-density measurements at $1400 \mathrm{MHz}$. These galaxies have jets classified as Fanaroff \& Riley (1974) type I (FR-I). HST imaging for the UGC FR-I sample is presented in Verdoes Kleijn et al. (1999, 2002). Xu et al. (2000) and Noel-Storr et al. (2003) report VLBA radio imaging and HST optical spectroscopy, respectively, for the sample.

We searched the literature for additional galaxies with FR-I or FR-I/II radio sources which show dust on HST imaging data. This resulted in 26 galaxies from the $3 \mathrm{CR}$ and $\mathrm{B} 2$ radio galaxy catalogues and one 4C galaxy. We refer to this extended sample as the FR sample, and list the general properties of the 47 FR-I galaxies in Table 1. The HST imaging data for 3CR and B2 galaxies are described in de Koff et al. (1996), Martel et al. (1999), Capetti et al. (2000) and Sparks et al. (2000) while the imaging for 4C-03.43 is described in Schmitt et al. (2002).

\subsection{The non-radio galaxy sample}

Figure 1 shows the location of the UGC FR-I sample members among the galaxies of the UGC in the plane of central stellar velocity dispersion versus host absolute magnitude. The data are taken from the LEDA catalogue ${ }^{1}$ except for the

\footnotetext{
${ }^{1}$ Lyon-Meudon Extragalactic Database (http://leda.univ-lyon1.fr).
} 
Table 1. Dust properties for the FR sample of radio galaxies. Properties of dust detected in the FR sample, which includes the 21 UGC FR-I radio galaxies as a subset (see Sect. 2). The latter are listed above the double horizontal lines. No dust is detected in two UGC FR-I galaxies: NGC 741 and NGC 2892. Column 1: target name. A* indicates radio sources which are sometimes classified as FR-Is (e.g., de Koff et al. 2000) and sometimes as FR-IIs (e.g., Chiaberge et al. 2002) or as intermediate, i.e., FR-I/II. Column 2: distance to the galaxy taken from Faber et al. 1989 or LEDA, except for NGC 5128 (Israel 1998). Column 3: dust morphology classification (see Sect. 3 for their definition). $1=$ dust lane, $2=$ dust ellipse, 3 = irregular dust. A 1-2 classification indicates an intermediate morphology classification: they could be either lanes or ellipses. Column 4: longest linear extent of the dust which has a typical relative error of $10 \%$. Entries marked with a ${ }^{1}$ have an additional irregular extended component. Column 5: ellipticity for dust ellipses. The error is given in between the brackets. We measured the ellipticity $\epsilon$ from the HST imaging except for 3C 402N, which was taken from Schmitt et al. (2002). Column 6: dust position angle with its error in between brackets. This is the PA of the major axis for dust ellipses and of the longest dust extent for dust lanes. An ${ }^{1}$ indicates that the dust feature was too faint to measure its position angle reliably. Column 7: the position angle difference between the dust and galaxy major axis. The error is listed in between brackets. A ${ }^{2}$ indicates that the galaxy is too round to determine its position angle. Column 8: position angle difference between jet axis and dust ellipse major axis or longest axis of the dust lane with the error in between brackets. Column 9: minimum misalignment angles $\theta_{\mathrm{DJ}}^{\min }$ between the jet axis and the normal of the dust disk. The first and second value are obtained assuming thin $(q=0)$ circular disks $(p=1)$ and elliptic disks $(p=0.75)$, respectively. Column 10: side of dust disk against which the main jet is projected; near/far indicates the side nearest or farthest from the observer, respectively. See Sect. 7.7 for details on its determination. Column 11: viewing angle to the main jet (i.e., the angle between the line of sight and the jet) as inferred from jet asymmetries at radio frequencies. This angle is taken from the literature (see references). Column 12: The jet misalignment angle as inferred from the viewing angle to the main jet. Column 13: reference for PA of jet and galaxy. 1: de Koff et al. (2000); 2: Martel et al. (1999); 3: Capetti et al. (2000); 4: Schmitt et al. (2002); 5: de Koff et al. (1996); 6: de Juan et al. (1996); 7: Guthrie (1980) (we measured the jet PA from Fig. 1); 8: Dufour et al. (1979); 9: Schreier et al. (1981); 10: VIMOS imaging (unpublished); 11: Verdoes Kleijn et al. (1999); 12: Verdoes Kleijn et al. (2002); 13: Leahy \& Perley (1991). References for $\theta_{\mathrm{JL}}$ : 14: Laing et al. (2004); 15: Laing et al. (1999); 16: Feretti et al. (1999); 17: Hardcastle et al. (1997).

\begin{tabular}{|c|c|c|c|c|c|c|c|c|c|c|c|c|}
\hline Target & $\begin{array}{c}D \\
(\mathrm{Mpc})\end{array}$ & Morph & $\begin{array}{l}\text { Size } \\
(\mathrm{pc})\end{array}$ & $\bar{\epsilon}$ & $\begin{array}{r}\mathrm{PA}_{\mathrm{D}} \\
\left(^{\circ}\right)\end{array}$ & $\begin{array}{r}\mathrm{PA}_{\mathrm{DG}} \\
\left({ }^{\circ}\right)\end{array}$ & $\begin{array}{r}\mathrm{PA}_{\mathrm{DJ}} \\
\left({ }^{\circ}\right)\end{array}$ & $\begin{array}{c}\theta_{\mathrm{DJ}}^{\min } \\
\left(^{\circ}\right)\end{array}$ & Side & $\begin{array}{l}\theta_{\mathrm{JL}} \\
\left({ }^{\circ}\right)\end{array}$ & $\begin{array}{r}\theta_{\mathrm{DJ}}^{\text {radio }} \\
\left(^{\circ}\right)\end{array}$ & "Ref. \\
\hline NGC 193 & 58 & 1 & $840^{1}$ & $\ldots$ & $0(6)$ & $58(7)$ & $77(10)$ & $\ldots$ & $\ldots$ & $\ldots$ & $\ldots$ & 11 \\
\hline NGC 315 & 68 & 2 & $830^{1}$ & $0.77(0.02)$ & $40(2)$ & $1(4)$ & $90(8)$ & $0 / 0$ & far & 37 & 40 & 11,14 \\
\hline NGC 383 & 65 & 2 & 2350 & $0.23(0.03)$ & $138(2)$ & $6(3)$ & $24(8)$ & $36 / 0$ & far & 52 & 47 & 11,14 \\
\hline NGC 541 & 73 & 2 & 640 & $0.09(0.05)$ & $\ldots$ & $\ldots$ & $\ldots$ & $\ldots$ & $\ldots$ & $\ldots$ & $\ldots$ & 11 \\
\hline UGC 1841 & 85 & $1-2$ & 330 & $<0.5$ & $\ldots$ & $\ldots$ & $\ldots$ & $\ldots$ & $\ldots$ & $\ldots$ & $\ldots$ & 11 \\
\hline NGC 2329 & 77 & 2 & 750 & $0.32(0.05)$ & $174(3)$ & $3(4)$ & $24(9)$ & $42 / 29$ & near & $\ldots$ & $\ldots$ & 11 \\
\hline NGC 3801 & 43 & 1 & $4430^{1}$ & $\ldots$ & $24(2)$ & $83(4)$ & $89(8)$ & $\ldots$ & $\ldots$ & $\ldots$ & $\ldots$ & 11 \\
\hline NGC 3862 & 84 & 2 & 610 & $0.01(0.02)$ & $\ldots$ & $\ldots$ & $\ldots$ & $\ldots$ & $\ldots$ & $\ldots$ & $\ldots$ & 11 \\
\hline UGC 7115 & 91 & 2 & 570 & $0.05(0.03)$ & $\ldots$ & $\ldots$ & $\ldots$ & $\ldots$ & $\ldots$ & $\ldots$ & $\ldots$ & 12 \\
\hline NGC 4261 & 30 & 2 & $240^{1}$ & $0.54(0.03)$ & $163(1)$ & $8(5)$ & $76(8)$ & $12 / 4$ & far & $\ldots$ & $\ldots$ & 11 \\
\hline NGC 4335 & 62 & 2 & $750^{1}$ & $0.59(0.10)$ & $158(2)$ & $2(5)$ & $79(8)$ & $10 / 3$ & $\ldots$ & $\ldots$ & $\ldots$ & 11 \\
\hline NGC 4374 & 15 & 1 & 990 & $\ldots$ & $79(10)$ & $49(11)$ & $80(13)$ & $\ldots$ & $\ldots$ & $\ldots$ & $\ldots$ & 11 \\
\hline NGC 4486 & 15 & 3 & 560 & $\ldots$ & $\ldots$ & $\ldots$ & $\ldots$ & $\ldots$ & $\ldots$ & $\ldots$ & $\ldots$ & 11 \\
\hline NGC 5127 & 64 & 1 & 950 & $\ldots$ & $48(2)$ & $20(5)$ & $70(8)$ & $\ldots$ & $\ldots$ & $\ldots$ & $\ldots$ & 11 \\
\hline NGC 5141 & 71 & 1 & 550 & $\ldots$ & $88(8)$ & $23(11)$ & $76(11)$ & $\ldots$ & $\ldots$ & $\ldots$ & $\ldots$ & 11 \\
\hline NGC 5490 & 70 & $1-2$ & 170 & $\gtrsim 0.5$ & $143(4)$ & $38(6)$ & $68(9)$ & $\ldots$ & $\ldots$ & $\ldots$ & $\ldots$ & 11 \\
\hline NGC 7052 & 55 & 2 & 1080 & $0.70(0.02)$ & $65(1)$ & 1(1) & $42(8)$ & $45 / 40$ & far & 79 & 47 & 11,15 \\
\hline UGC 12064 & 68 & 2 & 1200 & $0.54(0.05)$ & $166(5)$ & $6(5)$ & $25(9)$ & $54 / 43$ & near & 83 & 72 & 12,16 \\
\hline NGC 7626 & 47 & 1 & 230 & $\ldots$ & $175(4)$ & $4(6)$ & $57(9)$ & $\ldots$ & $\ldots$ & $\ldots$ & $\ldots$ & 11 \\
\hline NGC 5128 & 3.4 & 1 & 9200 & $>0.5$ & $120(3)$ & $85(4)$ & $67(9)$ & $\ldots$ & $\ldots$ & $\ldots$ & $\ldots$ & $8,9,10$ \\
\hline $3 \mathrm{C} 15^{*}$ & 292 & $1-2$ & 985 & $<0.5$ & 1 & $\ldots$ & $\ldots$ & $\ldots$ & $\ldots$ & $\ldots$ & $\ldots$ & 2 \\
\hline $3 C 29$ & 180 & $1-2$ & 486 & $<0.5$ & 1 & $\ldots$ & $\ldots$ & $\ldots$ & $\ldots$ & $\ldots$ & $\ldots$ & 2 \\
\hline $3 \mathrm{C} 40^{*}$ & 72 & 2 & 146 & $0.53(0.07)$ & $77(7)$ & $25(8)$ & $62(11)$ & $24 / 17$ & far & & & 2 \\
\hline $3 \mathrm{C} 75$ & 96 & $1-2$ & 194 & $>0.5$ & $36(5)$ & $65(5)$ & $76(9)$ & $\ldots$ & $\ldots$ & $\ldots$ & $\ldots$ & 2 \\
\hline 3C 76.1 & 128 & 2 & 892 & $0.65(0.05)$ & $39(4)$ & $89(5)$ & $72(9)$ & $17 / 11$ & $\ldots$ & $\ldots$ & $\ldots$ & 13 \\
\hline $3 \mathrm{C} 78$ & 116 & $1-2$ & 469 & $<0.5$ & 1 & $\ldots$ & $\ldots$ & $\ldots$ & $\ldots$ & $\ldots$ & $\ldots$ & 2 \\
\hline 3C 83.1 & 102 & 2 & 1020 & $0.86(0.03)$ & $168(2)$ & $2(4)$ & $82(8)$ & $8 / 6$ & $\ldots$ & $\ldots$ & $\ldots$ & 1,2 \\
\hline $3 C 84$ & 72 & 3 & $12060^{1}$ & $\ldots$ & $\ldots$ & $\ldots$ & $\ldots$ & $\ldots$ & $\ldots$ & $\ldots$ & $\ldots$ & 2 \\
\hline $3 \mathrm{C} 88^{*}$ & 121 & $1-2$ & 550 & $\lesssim 0.5$ & 1 & $\ldots$ & $\ldots$ & $\ldots$ & $\ldots$ & $\ldots$ & $\ldots$ & 1,2 \\
\hline
\end{tabular}


Table 1. continued.

\begin{tabular}{|c|c|c|c|c|c|c|c|c|c|c|c|c|}
\hline Target & $\begin{array}{c}D \\
(\mathrm{Mpc})\end{array}$ & Morph & $\begin{array}{l}\text { Size } \\
(\mathrm{pc})\end{array}$ & $\epsilon$ & $\begin{array}{r}\mathrm{PA}_{\mathrm{D}} \\
\left({ }^{\circ}\right)\end{array}$ & $\begin{array}{r}\Delta \mathrm{PA}_{\mathrm{DG}} \\
\left.\quad{ }^{\circ}\right)\end{array}$ & $\begin{array}{r}\Delta \mathrm{PA}_{\mathrm{DJ}} \\
\left({ }^{\circ}\right)\end{array}$ & $\begin{array}{c}\theta_{\mathrm{DJ}}^{\min } \\
\left({ }^{\circ}\right)\end{array}$ & Side & $\begin{array}{l}\theta_{\mathrm{JL}} \\
\left({ }^{\circ}\right)\end{array}$ & $\begin{array}{r}\theta_{\mathrm{DJ}}^{\text {radio }} \\
\left(^{\circ}\right)\end{array}$ & Ref. \\
\hline 3 C 296 & 95 & 2 & 568 & $0.71(0.05)$ & 157(4) & $12(4)$ & 60(9) & $29 / 23$ & far & $\leq 63$ & $30-73$ & $1,2,17$ \\
\hline 3C 315 & 433 & $1-2$ & 1480 & $\gtrsim 0.5$ & 1 & $\ldots$ & $\ldots$ & $\ldots$ & $\ldots$ & $\ldots$ & $\ldots$ & 1,5 \\
\hline 3C 317 & 140 & 3 & 11170 & $\ldots$ & $\ldots$ & $\ldots$ & $\ldots$ & $\ldots$ & $\ldots$ & $\ldots$ & $\ldots$ & 2 \\
\hline 3C 338 & 119 & 3 & 1750 & $\ldots$ & $\ldots$ & $\ldots$ & $\ldots$ & $\ldots$ & $\ldots$ & $\ldots$ & $\ldots$ & 1,2 \\
\hline $3 C 353^{*}$ & 122 & 1 & 1460 & $\ldots$ & $148(7)$ & 2 & $64(11)$ & $\ldots$ & $\ldots$ & $\ldots$ & $\ldots$ & 1,2 \\
\hline $3 \mathrm{C} 402 \mathrm{~N}$ & 101 & 2 & 1080 & $0.63(0.05)$ & $58(3)$ & $3(5)$ & 63(9) & $25 / 19$ & $\ldots$ & $\ldots$ & $\ldots$ & 4,6 \\
\hline $3 \mathrm{C} 442^{*}$ & 108 & 3 & 826 & $\ldots$ & $\ldots$ & $\ldots$ & $\ldots$ & $\ldots$ & $\ldots$ & $\ldots$ & $\ldots$ & 2 \\
\hline $3 C 452^{*}$ & 324 & $1-2$ & 3060 & $>0.5$ & $0(5)$ & $79(6)$ & $79(9)$ & $\ldots$ & $\ldots$ & $\ldots$ & $\ldots$ & 2 \\
\hline $4 C-03.43$ & 207 & $1-2$ & 450 & $\gtrsim 0.5$ & $86(5)$ & $68(6)$ & $69(9)$ & $\ldots$ & $\ldots$ & $\ldots$ & $\ldots$ & 4 \\
\hline B2 $0034+25$ & 128 & 2 & 1210 & $0.72(0.06)$ & $160(4)$ & $0(4)$ & $67(9)$ & $22 / 17$ & far & 64 & 24 & 3,15 \\
\hline B2 0915+32 & 248 & 2 & 1540 & $0.28(0.04)$ & $122(10)$ & $22(11)$ & $88(13)$ & $1 / 0$ & near & 80 & 56 & 3,15 \\
\hline B2 $1256+28$ & 90 & 2 & 360 & $0.81(0.10)$ & $178(3)$ & $4(4)$ & $43(8)$ & $46 / 43$ & $\ldots$ & $\ldots$ & $\ldots$ & 3,7 \\
\hline B2 $1339+26$ & 303 & $1-2$ & 580 & $\sim 0.5$ & $0(10)$ & $13(10)$ & $30(13)$ & $\ldots$ & $\ldots$ & $\ldots$ & $\ldots$ & 3 \\
\hline B2 $1346+26$ & 253 & 1 & $6710^{1}$ & $\ldots$ & $134(7)$ & $62(11)$ & $71(11)$ & $\ldots$ & $\ldots$ & $\ldots$ & $\ldots$ & 3 \\
\hline B2 $1357+28$ & 251 & $1-2$ & 520 & $\gtrsim 0.5$ & $95(5)$ & $2(10)$ & $85(9)$ & $\ldots$ & $\ldots$ & $\ldots$ & $\ldots$ & 3 \\
\hline B2 $1457+29$ & 588 & $1-2$ & 5000 & $\gtrsim 0.5$ & $36(4)$ & $78(5)$ & 61(9) & $\ldots$ & $\ldots$ & $\ldots$ & $\ldots$ & 3 \\
\hline B2 $1525+29$ & 261 & $1-2$ & 540 & $\gtrsim 0.5$ & $148(5)$ & $28(5)$ & $62(9)$ & $\ldots$ & $\ldots$ & $\ldots$ & $\ldots$ & 3 \\
\hline B2 $2335+26$ & 120 & 2 & 1160 & $0.25(0.05)$ & $6(9)$ & 24(9) & $61(12)$ & $19 / 0$ & far & $\ldots$ & $\ldots$ & 3 \\
\hline
\end{tabular}

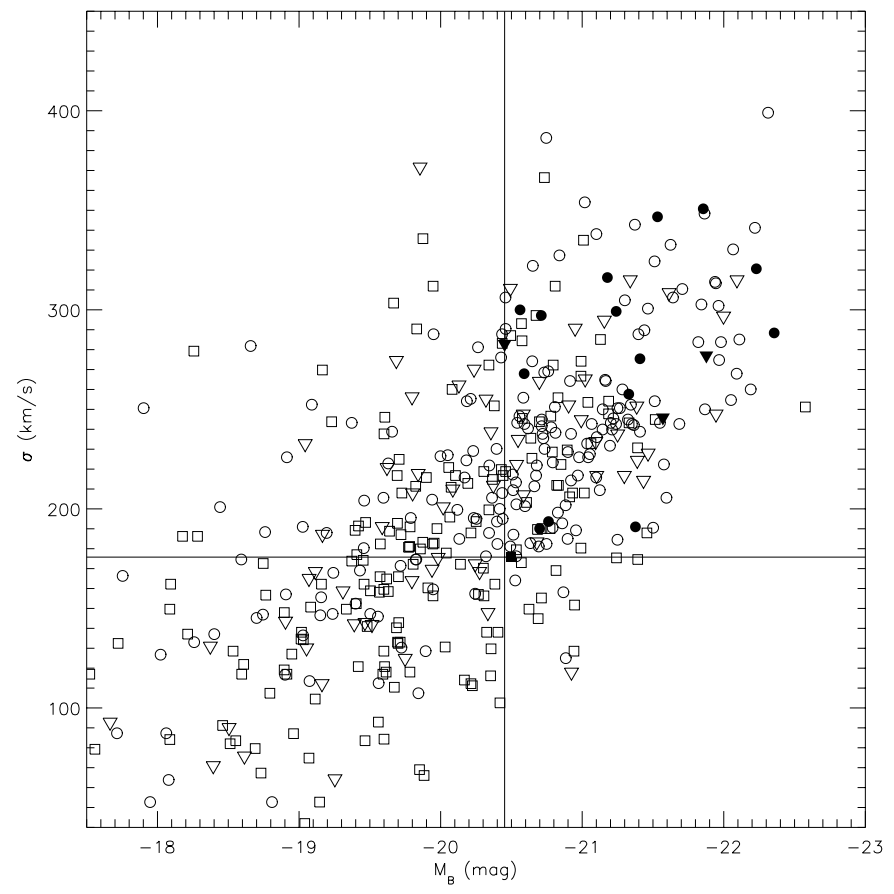

Fig. 1. Central stellar velocity dispersion $\sigma$ versus total absolute blue magnitude $M_{B}$ for early-type galaxies in the Uppsala General Catalogue at $v<7000 \mathrm{~km} \mathrm{~s}^{-1}$. Circles indicate ellipticals, triangles are $\mathrm{E} / \mathrm{S} 0$ transition objects and squares denote $\mathrm{S} 0$ s. The filled symbols indicate the 18 radio galaxies of the UGC FR-I sample for which a $\sigma$ has been published. The horizontal and vertical solid lines mark the minimum $\sigma$ and galaxy luminosity observed for the radio galaxies. Magnitudes, dispersions and morphologies are taken from the LEDA catalogue.

velocity dispersion of 3C 66B and 3C 449 (Balcells et al. 1995) and NGC 4335 (Verdoes Kleijn et al. 2002). The location of the UGC FR-I galaxies confirms the well-known fact that radio galaxies are bright early-type galaxies. The figure also shows that the FR-I galaxies are distributed fairly uniformly, relative to the general galaxy distribution, in the upper-right corner of large host magnitude and velocity dispersion. The morphologies of the UGC FR-I galaxies are not a random selection from the morphology distribution in this upper-right corner. Table 2 shows that the UGC FR-I galaxies are predominantly ellipticals and $\mathrm{E} / \mathrm{S} 0$ transition objects and rarely pure SOs.

To carry out a statistical comparison of the dust distributions in early-type galaxies with and without radio jets, we need samples of both classes of galaxies with similar global properties of their stellar host and similar HST imaging of the central regions so that we can perform an identical dust analysis. Ideally, one would like to perform the dust analysis for a welldefined sample of quiescent galaxies in the UGC catalogue from which also the radio galaxies were selected. Tran et al. (2001) analysed dust features in a representative mostly quiescent sample of nearby $\left(v<3400 \mathrm{~km} \mathrm{~s}^{-1}\right)$ early-type galaxies using HST/WFPC2 observations. Unfortunately, this sample contains galaxies with a typical host magnitude $\sim 2$ mag fainter than in the UGC FR-I sample. It also has a fraction of lenticular galaxies which is about four times higher. We therefore crosscorrelated the HST/- WFPC2 archive with the UGC catalogue to select all early-type UGC galaxies at $v<7000 \mathrm{~km} \mathrm{~s}^{-1}$ which have a similar absolute magnitude as the radio galaxies (i.e., $M_{B}<-20.4^{\mathrm{m}}$ ), but which are not part of the UGC FR-I sample. The resulting sample of 52 galaxies is listed in Table 3 and its general properties are shown in Fig. 2. Some of the non-FR-I galaxies host low-level activity in their nuclei, e.g., nuclear radio emission and optical line-emission. For clarity we therefore refer to this sample as the UGC non-FR-I sample instead of, for example, the quiescent sample. However, none of the UGC non-FR-I galaxies have jets on scales of tens 
Table 2. Host morphologies. Relative distribution over host morphology (taken from the LEDA catalogue) for the UGC, UGC FR-I, UGC non-FR-I samples of galaxies. Column 2: total number of galaxies in the sample. Columns3-5: the fraction of galaxies within each sample for the three morphology classes and the Poissonian error.

\begin{tabular}{llllll}
\hline \hline Sample & $N$ & $E$ & $E /$ SO & SO & Comments \\
\hline UGC total & 1101 & $0.34 \pm 0.02$ & $0.16 \pm 0.01$ & $0.50 \pm 0.02$ & $v<7000 \mathrm{~km} \mathrm{~s}^{-1}$ \\
& 389 & $0.49 \pm 0.04$ & $0.17 \pm 0.02$ & $0.34 \pm 0.03$ & $v<7000 \mathrm{~km} \mathrm{~s}^{-1}$ and $M_{B}<-20.4$ \\
UGC FR-I & 21 & $0.71 \pm 0.18$ & $0.19 \pm 0.10$ & $0.10 \pm 0.07$ & \\
UGC non-FR-I & 52 & $0.71 \pm 0.11$ & $0.12 \pm 0.05$ & $0.17 \pm 0.06$ & \\
\hline
\end{tabular}
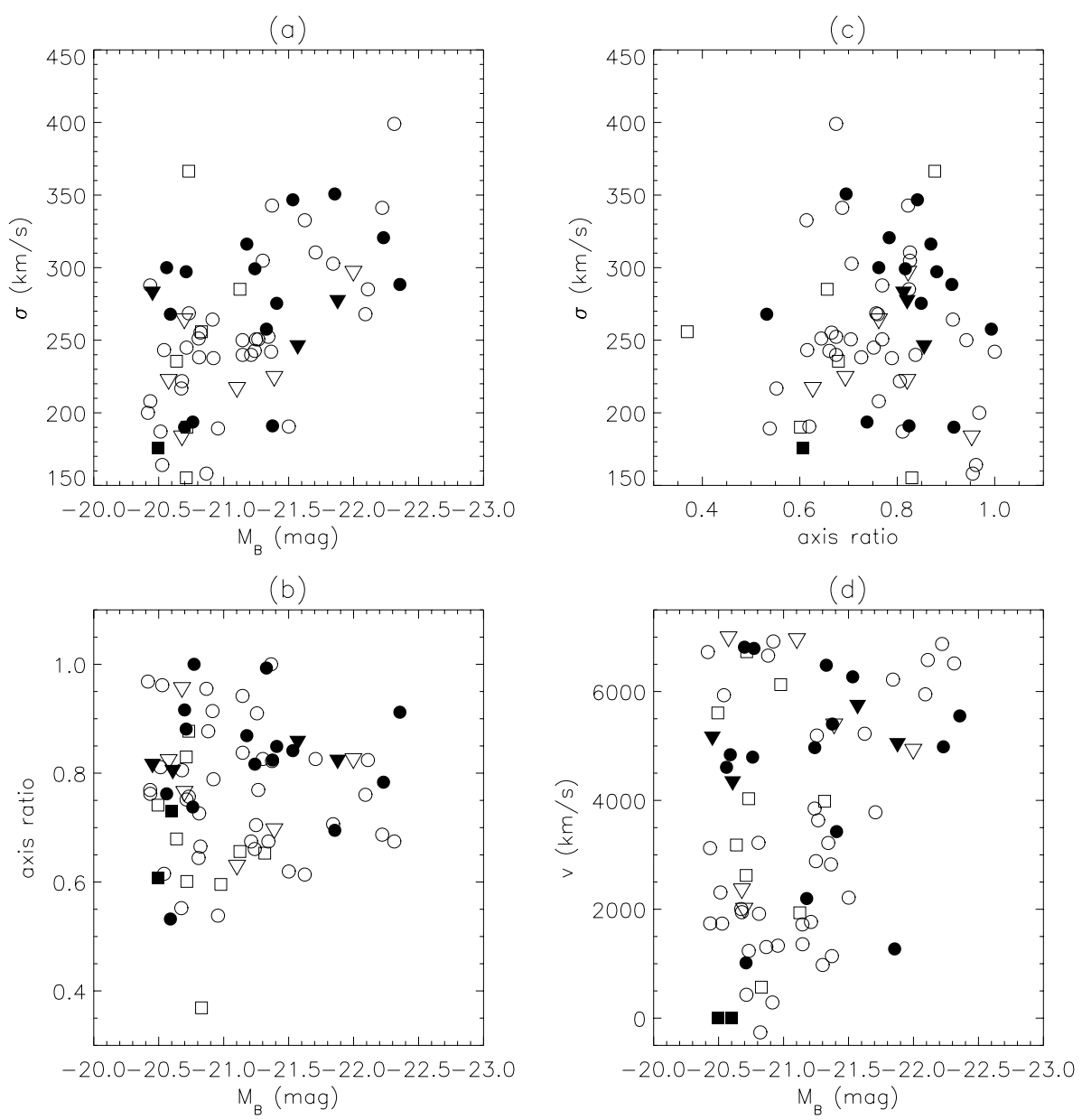

Fig. 2. Central stellar velocity dispersion $\sigma$, host absolute blue magnitude $M_{B}$, isophotal axis ratio (at a blue surface brightness of $25 \mathrm{mag} / \mathrm{arcsec}^{-2}$ ) and recession velocity $V$ for the UGC non-FR-I sample of early-type galaxies (open symbols) and the UGC FR-I radio galaxies (filled symbols). Circles indicate ellipticals, triangles E/S0 are transition objects and squares denote S0s.

of kiloparsec or larger. The UGC non-FR-I sample turns out to match the UGC FR-I sample in velocity dispersion, recession velocity and morphologies (Fig. 2 and Table 2).

The UGC FR-I and UGC non-FR-I samples also match in imaging depth and filter coverage. The entire UGC FR-I sample was observed in $V$ - and $I$-band. Table 3 shows that $88 \%$ of the galaxies has $V$ and/or $I$-band observations, 54\% has $V$ - and $I$-band observations and $12 \%$ has only $R$-band observations. Thus the filter coverage is similar for both samples. The many targets with both $V$ - and $I$-band observations allow us to verify that the dust detection rate and the derived dust properties do not depend on the filter used. For the UGC FRI sample the exposure times for both $V$ - and $I$-band images are typically $460 \mathrm{~s}$ (increasing up to $1400 \mathrm{~s}$ in a small subset of the observations). Table 3 shows that the exposure times for the UGC non-FR-I galaxies are similar.

\section{Dust properties and position angle measurements}

It is likely that every early-type galaxy contains dust due to mass loss from evolved stars. In addition, it can contain externally acquired dust from tidal interactions or accretion of galaxy satellites. Whether the dust obscuration is detected in optical observations depends on various factors, including sensitivity of the observations, dust morphology (e.g., smooth 
Table 3. UGC non-FR-I sample. General properties of the 52 UGC non-FR-I galaxy sample which forms the comparison sample for the UGC FR-I sample of radio galaxies. Galaxy data are taken from the LEDA catalogue. Columns 1-3: galaxy name, Hubble type and absolute blue magnitude. Columns 4-5: WFPC2 filter name and exposure time. Column 6: HST program number.

\begin{tabular}{|c|c|c|c|c|c|c|c|c|c|c|c|}
\hline NGC & $\begin{array}{c}\text { Hubble } \\
\text { type }\end{array}$ & $\begin{array}{c}M_{B} \\
(\mathrm{mag})\end{array}$ & Filters & $\begin{array}{r}\text { Time } \\
(\mathrm{s})\end{array}$ & $\begin{array}{c}\text { HST } \\
\text { program }\end{array}$ & NGC & $\begin{array}{c}\text { Hubble } \\
\text { type }\end{array}$ & $\begin{array}{c}M_{B} \\
(\mathrm{mag})\end{array}$ & Filters & $\begin{array}{r}\text { Time } \\
(\mathrm{s})\end{array}$ & $\begin{array}{c}\text { HST } \\
\text { program }\end{array}$ \\
\hline 507 & E-S0 & -22.0 & $F 555 W$ & 1600 & 6587 & 4494 & $\mathrm{E}$ & -21.0 & $F 555 W$ & 1000 & 5454 \\
\hline 545 & E-S0 & -21.4 & $F 814 W$ & 1000 & 8683 & & & & $F 814 W$ & 460 & 5454 \\
\hline \multirow[t]{2}{*}{821} & $\mathrm{E}$ & -20.4 & $F 555 W$ & 700 & 6099 & 4526 & So & -20.8 & $F 555 W$ & 520 & 5375 \\
\hline & & & $F 814 W$ & 460 & 6099 & & & & $F 814 W$ & 520 & 5375 \\
\hline 910 & $\mathrm{E}$ & -21.8 & $F 814 W$ & 1000 & 8683 & 4552 & E-S0 & -20.9 & F555W & 2400 & 6099 \\
\hline 1016 & E & -22.5 & F555W & 1600 & 6587 & & & & $F 814 W$ & 1500 & 6099 \\
\hline \multirow[t]{2}{*}{1129} & So & -22.2 & $F 555 W$ & 6500 & 6810 & 4589 & E & -21.0 & $F 555 W$ & 1000 & 5454 \\
\hline & & & $F 814 W$ & 6500 & 6810 & & & & $F 814 W$ & 460 & 5454 \\
\hline 1161 & E-S0 & -21.3 & $F 547 M$ & 360 & 6837 & 4621 & E & -20.7 & $F 555 W$ & 1050 & 5512 \\
\hline \multirow[t]{2}{*}{1497} & So & -21.0 & $F 547 M$ & 300 & 5924 & & & & $F 814 W$ & 1050 & 5512 \\
\hline & & & $F 791 W$ & 100 & 5924 & 4649 & E & -21.5 & F555W & 2100 & 6286 \\
\hline UGC 3426 & So & -20.7 & $F 814 W$ & 260 & 8645 & & & & $F 814 W$ & 2500 & 6286 \\
\hline 2258 & So & -21.3 & $F 814 W$ & 2700 & 8212 & 4807 & E-S0 & -20.7 & F606W & 400 & 5997 \\
\hline \multirow[t]{2}{*}{2300} & $\mathrm{E}$ & -20.9 & $F 555 W$ & 1520 & 6099 & 4816 & E-S0 & -21.4 & $F 606 W$ & 800 & 5997 \\
\hline & & & $F 814 W$ & 1450 & 6099 & 4881 & E & -20.7 & F555W & 4000 & 5233 \\
\hline \multirow[t]{2}{*}{2768} & E-S0 & -21.1 & $F 555 W$ & 1000 & 6587 & & & & $F 814 W$ & 4000 & 5233 \\
\hline & & & $F 814 W$ & 2000 & 6587 & 4889 & $\mathrm{E}$ & -22.6 & F606W & 320 & 5997 \\
\hline 2832 & E & -22.4 & $F 814 W$ & 2600 & 8184 & 4952 & E & -21.6 & $F 606 W$ & 400 & 5997 \\
\hline 2872 & E & -20.5 & $F 702 W$ & 1000 & 6357 & 4957 & E & -21.2 & F606W & 400 & 5997 \\
\hline 2911 & E-S0 & -20.8 & $F 547 M$ & 460 & 5924 & 5252 & So & -21.0 & F606W & 500 & 5479 \\
\hline 3348 & E & -21.4 & $F 702 \mathrm{~W}$ & 1000 & 6357 & 5322 & E & -21.4 & F555W & 1000 & 5454 \\
\hline \multirow[t]{2}{*}{3516} & So & -20.9 & $F 555 W$ & 1000 & 6633 & & & & $F 814 W$ & 460 & 5454 \\
\hline & & & $F 814 W$ & 730 & 6633 & 5557 & E & -21.7 & $F 555 W$ & 1000 & 6587 \\
\hline \multirow[t]{2}{*}{3610} & $\mathrm{E}$ & -20.8 & $F 555 W$ & 1000 & 6587 & 5813 & E & -21.0 & $F 555 W$ & 1000 & 5454 \\
\hline & & & $F 814 W$ & 2000 & 6587 & & & & $F 814 W$ & 460 & 5454 \\
\hline 3613 & E & -20.9 & $F 702 W$ & 1000 & 6357 & 5846 & E & -21.2 & F555W & 2200 & 5920 \\
\hline 3842 & E & -22.2 & $F 555 W$ & 1600 & 6587 & & & & $F 814 W$ & 2300 & 5920 \\
\hline 3894 & E-S0 & -21.0 & $F 547 M$ & 503 & 5924 & 5982 & E & -21.5 & F555W & 1000 & 5454 \\
\hline 4073 & E & -22.4 & $F 555 W$ & 1600 & 6587 & & & & $F 814 W$ & 460 & 5454 \\
\hline \multirow[t]{2}{*}{4125} & E & -21.3 & F555W & 1000 & 6587 & 6211 & So & -20.9 & F606W & 500 & 5479 \\
\hline & & & $F 814 W$ & 2000 & 6587 & 6703 & E-S0 & -20.8 & $F 814 W$ & 320 & 5999 \\
\hline \multirow[t]{2}{*}{4168} & $\mathrm{E}$ & -20.7 & $F 547 M$ & 460 & 6837 & $7318 \mathrm{~A}$ & $\mathrm{E}$ & -20.9 & $F 569 W$ & 1600 & 6596 \\
\hline & & & $F 702 \mathrm{~W}$ & 1000 & 6357 & & & & $F 814 W$ & 1000 & 6596 \\
\hline \multirow[t]{2}{*}{4365} & $\mathrm{E}$ & -20.9 & $F 555 W$ & 1000 & 5454 & 7562 & E & -21.1 & F555W & 2200 & 6554 \\
\hline & & & $F 814 W$ & 460 & 5454 & & & & $F 814 W$ & 2200 & 6554 \\
\hline \multirow[t]{2}{*}{4406} & $\mathrm{E}$ & -20.8 & F555W & 1000 & 5454 & 7619 & E & -21.8 & F555W & 2200 & 6554 \\
\hline & & & $F 814 W$ & 460 & 5454 & & & & $F 814 W$ & 2200 & 6554 \\
\hline \multirow[t]{2}{*}{4472} & $\mathrm{E}$ & -21.4 & $F 555 W$ & 460 & 6673 & 7785 & E & -21.3 & $F 555 W$ & 800 & 6587 \\
\hline & & & $F 814 W$ & 460 & 6673 & & & & & & \\
\hline \multirow[t]{2}{*}{4473} & $\mathrm{E}$ & -21.6 & $F 555 W$ & 1800 & 6099 & & & & & & \\
\hline & & & $F 814 W$ & 2000 & 6099 & & & & & & \\
\hline
\end{tabular}

or clumpy) and orientation of dust with respect to the observer (e.g., face-on or edge-on). To ensure a homogeneous dust classification and measurement of dust properties for all samples we re-analysed the published HST imaging using the HST archive. We define "dust" as a localized depression in the stellar surface brightness. Thus we are insensitive to a global smooth dust component (e.g., following the stellar distribution) if it were present in early-type galaxies. Our approach is similar to that used in earlier HST imaging studies (e.g., van Dokkum \& Franx 1995; Verdoes Kleijn et al. 1999; Tran et al. 2001). 
The appearance of the dust structures in both radio and nonradio galaxies varies from regular elliptical shapes, evoking the idea of inclined disks, to highly irregular structures, suggesting unsettled dust. We use the classification scheme for the UGC FR-I sample described in Verdoes Kleijn et al. (1999). It divides the morphology of the dust structure as projected on the plane of the sky into three bins. Class one, a "dust lane", is a filamentary structure which passes through the centre and which is sufficiently regular to assign an orientation to it. Class two, a "dust ellipse", is a dust structure with a circumference that resembles an ellipse. In some galaxies it is not possible to determine unambiguously if the dust has an ellipse- or a lane-like appearance. This is often due to the faintness and/or rather small angular size of the dust distribution. We classify these galaxies as "intermediate" (see Tables 1 and 4). Class three, "irregular dust", is extended clumpy and/or filamentary dust which either is too irregular to assign an orientation or does not pass through the nucleus and hence is not classified as a lane. Some galaxies which host a dust ellipse or lane also harbour a more extended, class three, irregular dust distribution (see Tables 1 and 4). We discuss in Sects. 6 and 9 how the dust classification scheme, based on appearance, depends on viewing angle towards the dust distribution.

We define the size of the dust as the largest linear extent of the dust feature. This is the dust major axis for dust ellipses. We measure the ellipticity $\epsilon$ of dust ellipses using their circumference. Dust classes one and two, the lanes and ellipses, are regular and extended enough to define also a position angle $\mathrm{PA}_{\mathrm{D}}$. For lanes it is the PA of the filament, taken as close as possible to the nucleus if any bending is present. For ellipses it is the PA of the dust ellipse major axis. For close-to-round ellipses $(\epsilon<0.1)$ we cannot determine a reliable PA of the major axis. The dust classification and dust properties for the FR-I and UGC non-FR-I samples are listed in Tables 1 and 4, respectively. As a cross-check we compared our measurements of dust position angle and ellipticities with measurements available in the literature for various galaxies (Table 5). Ellipticities differ by $\lesssim 0.05$ and $\mathrm{PA}_{\mathrm{D}}$ differ by $\lesssim 10^{\circ}$.

We define the galaxy orientation as the position angle of the stellar isophotal major axis just outside the radius of the main dust distribution. For the UGC non-FR-I, the B2 and some 3CR galaxies, we measured the PA ourselves from isophotal fitting to the WFPC2 images, masking dusty regions. The PA was taken from the literature for the remaining radio galaxies (see Table 1 for references).

For the radio galaxies we also require the position angle $\mathrm{PA}_{J}$ of the jet axis close to the galaxy centre. For the UGC FR-I sample, we use the $\mathrm{PA}_{\mathrm{J}}$ as determined by $\mathrm{Xu}$ et al. (2000). These PAs are obtained from VLBA measurements, or, if those are not available, from the larger-scale VLA measurements. In cases where both VLA and VLBA measurements are available, the difference in $\mathrm{PA}_{\mathrm{J}}$ is $4^{\circ}$ on average and always $\leq 14^{\circ}$. For the other radio galaxies the $\mathrm{PA}_{\mathrm{J}}$ was taken from various studies (see Table 1 for references). Given the difference in PA $A_{J}$ between VLA and VLBA measurements and the published values for other jets, we estimate a typical error of $8^{\circ}$ for all $\mathrm{PA}_{\mathrm{J}}$.
Table 4. Dust properties UGC non-FR-I sample. Properties of the dust detected in UGC non-FR-I sample galaxies. Column 2: galaxy distance from Faber et al. (1989) or LEDA, except for NGC 4526, which is taken from Tonry et al. (2001). Column 3: dust morphology (see Sect. 3 for definitions). 1 = dust lane, $2=$ dust ellipse, $1-2=$ either lane or ellipse and $3=$ irregular dust. Column 4 : longest linear extent of the dust which has a typical relative error of $10 \%$. Entries marked with a ${ }^{1}$ have an additional irregular extended component. Column 5: ellipticity of dust ellipses, with the measurement error in between brackets. Column 6: dust position angle and its error in between brackets. This is the PA of the major axis for dust ellipses and of the longest dust extent for dust lanes. Column 7: position angle difference between the galaxy major axis and dust longest axis and its error in between brackets (see Sect. 3).

\begin{tabular}{lccccrr}
\hline \hline NGC & $\begin{array}{c}D \\
(\mathrm{Mpc})\end{array}$ & morph & $\begin{array}{r}\text { size } \\
(\mathrm{pc})\end{array}$ & $\epsilon$ & $\begin{array}{r}\mathrm{PA}_{\mathrm{D}} \\
\left({ }^{\circ}\right)\end{array}$ & $\begin{array}{r}\Delta \mathrm{PA}_{\mathrm{DG}} \\
\left({ }^{\circ}\right)\end{array}$ \\
\hline 910 & 69.23 & $1-2$ & 160 & 20.5 & $\ldots$ & $\ldots$ \\
1129 & 69.67 & 2 & 670 & $0.85(0.10)$ & $0(2)$ & $0(3)$ \\
1161 & 25.80 & 2 & 2960 & $0.53(0.05)$ & $135(5)$ & $2(7)$ \\
1497 & 81.69 & 3 & 5650 & $\ldots$ & $\ldots$ & $\ldots$ \\
2258 & 53.12 & 3 & 470 & $\ldots$ & $\ldots$ & $\ldots$ \\
2768 & 20.43 & 1 & $120^{1}$ & $\ldots$ & $53(8)$ & $84(9)$ \\
2872 & 41.65 & 2 & 150 & $0.46(0.06)$ & $171(5)$ & $2(5)$ \\
2911 & 42.40 & 1 & $1080^{1}$ & $\ldots$ & $63(5)$ & $85(11)$ \\
$\mathrm{UGC} 3426$ & 53.73 & 3 & $3040^{1}$ & $\ldots$ & $\ldots$ & $\ldots$ \\
3516 & 34.95 & 3 & 2290 & $\ldots$ & $\ldots$ & $\ldots$ \\
3894 & 42.95 & 1 & $1490^{1}$ & $\ldots$ & $109(4)$ & $14(4)$ \\
4125 & 26.48 & 1 & $170^{1}$ & $\ldots$ & $108(10)$ & $13(18)$ \\
4406 & 17.77 & $1-2$ & 50 & $\lesssim 0.5$ & $\ldots$ & $\ldots$ \\
4472 & 17.77 & 1 & 210 & $\ldots$ & $136(10)$ & $26(11)$ \\
4494 & 9.267 & 2 & 70 & $0.50(0.05)$ & $23(6)$ & $1(6)$ \\
4526 & 16.90 & 2 & 2350 & $0.80(0.05)$ & $127(5)$ & $5(7)$ \\
4552 & 17.77 & $1-2$ & $40^{1}$ & $\leq 0.5$ & $\ldots$ & $\ldots$ \\
4589 & 40.40 & 1 & $2310^{1}$ & $\ldots$ & $171(10)$ & $89(11)$ \\
4952 & 79.11 & 2 & 190 & $0.54(0.03)$ & $30(5)$ & $2(5)$ \\
5252 & 89.75 & 3 & 2510 & $\ldots$ & $\ldots$ & $\ldots$ \\
5322 & 22.15 & 2 & 370 & $0.87(0.05)$ & $88(5)$ & $2(5)$ \\
5813 & 31.15 & 3 & 1690 & $\ldots$ & $\ldots$ & $\ldots$ \\
5846 & 31.15 & 3 & 2840 & $\ldots$ & $\ldots$ & $\ldots$ \\
$7318 \mathrm{~A}$ & 88.80 & 3 & 3790 & $\ldots$ & $\ldots$ & $\ldots$ \\
7785 & 60.05 & 3 & 1610 & $\ldots$ & $\ldots$ & $\ldots$ \\
\hline & & & & & &
\end{tabular}

We are interested in the relative orientation of host, dust and jet if present. Thus we list in Tables 1 and 4 the position angle difference $\triangle \mathrm{PA} A_{\mathrm{DG}}$ between dust and galaxy axis and $\triangle \mathrm{PA} \mathrm{DJ}_{\mathrm{DJ}}$ between dust and jet axis, both defined in the range $\left[0^{\circ}, 90^{\circ}\right]$.

\section{Comparison of dust properties}

We first check whether the observed dust properties in our galaxy samples depend on distance. Figure 3 compares the radio galaxies (the UGC FR-I sample) and non-radio galaxies (the UGC non-FR-I sample). The top diagram shows that galaxies with and without dust detections are observed throughout the sampled volume. A similar range in dust sizes is 
Table 5. Comparison between published and our measurements of dust ellipticities and PAs. Columns 2-3: ellipticity as determined by us with the error in between brackets and as reported in the literature, respectively. Columns 4-5: position angle of the dust as determined by us (with error again between the brackets) and as reported in the literature, respectively. Column 8: references 1: de Koff et al. (2000); 2: Capetti \& Celotti (1999); 3: Capetti et al. (2000); 4: Schmitt et al. (2002); 5: van der Marel \& van den Bosch (1998); 6: de Ruiter et al. (2002); 7: Dufour et al. (1979). The reason for the significant difference in the $\mathrm{PA}_{\mathrm{D}}$ for $\mathrm{B} 21346+26$, B2 $1525+29$ as measured by de Ruiter et al. (2002) and us on the one hand and by Capetti et al. (2000) on the other hand is unknown, but the de Ruiter et al. (2002) measurements are deemed more accurate (Capetti private comm.).

\begin{tabular}{lrrrrr}
\hline \hline Name & $\epsilon$ & $\epsilon_{\text {ref }}$ & $\begin{array}{r}\mathrm{PA}_{\mathrm{D}} \\
\left({ }^{\circ}\right)\end{array}$ & $\begin{array}{r}\mathrm{PA}_{\text {ref }} \\
\left({ }^{\circ}\right)\end{array}$ & Ref. \\
\hline NGC 383 & $0.23(0.03)$ & 0.18 & $138(2)$ & 135 & 2 \\
NGC 4261 & $0.54(0.03)$ & 0.58 & $163(1)$ & 165 & 2 \\
NGC 7052 & $0.70(0.02)$ & 0.65 & $65(1)$ & 65 & 5 \\
3C 449 & $0.54(0.05)$ & 0.50 & $166(5)$ & 169 & 1 \\
NGC 5128 & $\ldots$ & $\ldots$ & $120(3)$ & 122 & 7 \\
3C 83.1 & $0.86(0.03)$ & 0.91 & $168(2)$ & 171 & 1 \\
3C 296 & $0.71(0.05)$ & 0.71 & $157(4)$ & 160 & 1 \\
3C 353 & $\ldots$ & $\ldots$ & $148(7)$ & 166 & 1 \\
3C 402N & $\ldots$ & $\ldots$ & $58(3)$ & 55 & 4 \\
4C-03.43 & $\ldots$ & $\ldots$ & $86(5)$ & 73 & 4 \\
B2 0034+25 & $0.72(0.06)$ & $\ldots$ & $160(4)$ & $160 / 160$ & $3 / 6$ \\
B2 0915+32 & $0.28(0.04)$ & $\ldots$ & $122(10)$ & 125 & 6 \\
B2 1256+28 & $0.81(0.10)$ & $\ldots$ & $178(3)$ & 0 & 6 \\
B2 1339+26 & $\ldots$ & $\ldots$ & $0(10)$ & $0 / 175$ & $3 / 6$ \\
B2 1346+26 & $\ldots$ & $\ldots$ & $134(7)$ & $0 / 134$ & $3 / 6$ \\
B2 1357+28 & $\ldots$ & $\ldots$ & $95(5)$ & $95 / 77$ & $3 / 6$ \\
B2 1457+29 & $\ldots$ & $\ldots$ & $36(4)$ & $30 / 50$ & $3 / 6$ \\
B2 1525+29 & $\ldots$ & $\ldots$ & $148(5)$ & $25 / 144$ & $3 / 6$ \\
B2 2335+26 & $0.25(0.05)$ & $\ldots$ & $6(9)$ & 8 & 6 \\
\hline
\end{tabular}

detected at every distance except for small $(<100 \mathrm{pc})$ dust distributions which HST can resolve well only at small distances. We see no trend in morphology classification with distance. We conclude that the detection of dust, the classification and its size do not correlate with galaxy distance for $v<7000 \mathrm{~km} \mathrm{~s}^{-1}$.

We detect dust in $48 \% \pm 10 \%$ of the UGC non-FR-I galaxies. This detection rate is similar to the $40 \% \pm 9 \%$ and $43 \% \pm$ $8 \%$ detection rates from HST imaging for the samples of nearby early-type galaxies as compiled by van Dokkum \& Franx (1995) - excluding galaxies with extended radio structures in their sample - and by Tran et al. (2001), respectively. Both studies report an increased dust detection rate for galaxies with radio emission (compact and/or extended), but those rates are still less than the $90 \% \pm 7 \%$ detection rate for our UGC FR-I sample of more powerful radio galaxies. At much lower radio luminosities $\left(L \sim 10^{19-21} \mathrm{~W} / \mathrm{Hz}\right.$ at $\left.3.6 \mathrm{~cm}\right)$, the radio luminosity functions of nearby early-type galaxies with and without HST dust detection seem to become very similar (Krajnović \& Jaffe 2002). Hence, there is an increasing dust detection rate with increasing radio power. Many of the dusty non-radio galaxies have low-level active galactic nuclei,

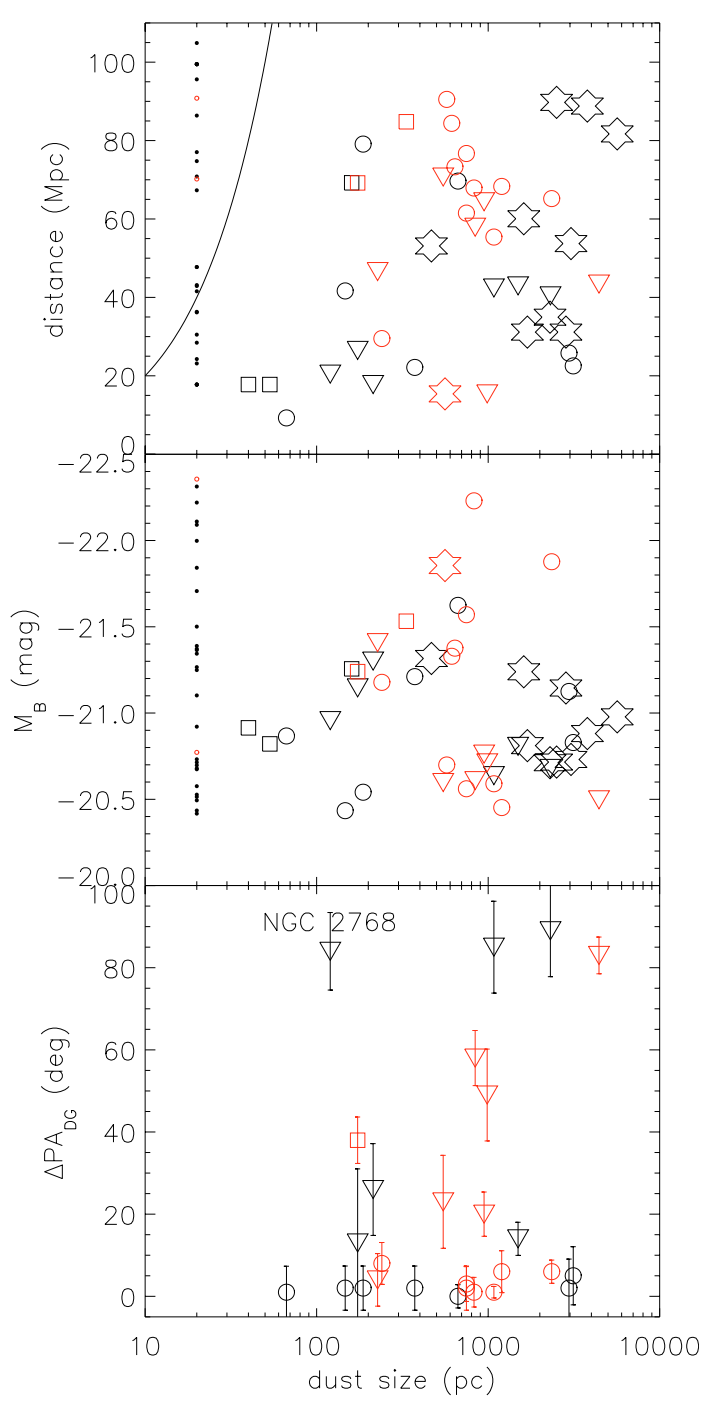

Fig. 3. From top to bottom: galaxy distance, absolute blue magnitude and position angle difference $\triangle \mathrm{PA} \mathrm{DG}_{\mathrm{D}}$ between host galaxy and central dust as a function of linear dust extent. UGC FR-I galaxies are represented by red symbols and UGC non-FR-I galaxies by black symbols. Circles indicate dust ellipses, triangles indicate dust lanes, squares indicate dust structures which have a morphology in between ellipse and lane and stars indicate irregular dust (see Sect. 3). The small dots indicate galaxies without dust detection plotted with a fiducial linear dust extent of $20 \mathrm{pc}$. The typical relative error on dust size is $10 \%$. The curve in the top panel indicates the minimum linear dust extent which can be resolved as a function of distance. In the bottom panel NGC 2768 is indicated because it has irregular dust with an extent of $\sim 1.7 \mathrm{kpc}$ roughly parallel to its small scale dust lane. See Sect. 4 for further discussion.

sometimes with radio cores (e.g., Ho et al. 1997; Tran et al. 2001). Thus the difference in detection rate is perhaps a trivial effect: it could reflect the simple fact that all radio galaxies have active galactic nuclei. The close connection between dust and nuclear activity then supports the idea that the central dusty ISM forms - not surprisingly - the fuel reservoir for the active nucleus.

All three dust morphologies occur in both radio and non-radio galaxies. Dust features with a lane-like morphology appear in dusty radio and non-radio galaxies at similar 
Table 6. Fractions of dust morphologies. Frequency of dust morphologies as a fraction of total number of dust galaxies for the UGC FR-I and UGC non-FR-I sample. Columns 2-5 list the fractions of dust lanes, ellipses, dust structures which are either ellipses or lanes and irregular dust respectively. The definitions of these morphology classes are given in Sect. 4.

\begin{tabular}{lcccc}
\hline \hline Sample & Lanes & Ellipses & Ambiguous & Irregular \\
\hline UGC non-FR-I & $0.24 \pm 0.10$ & $0.28 \pm 0.11$ & $0.12 \pm 0.07$ & $0.36 \pm 0.12$ \\
UGC FR-I & $0.32 \pm 0.13$ & $0.53 \pm 0.17$ & $0.10 \pm 0.07$ & $0.05 \pm 0.05$ \\
\hline
\end{tabular}

frequencies. In contrast, irregular dust distributions are more often seen in non-radio galaxies while radio galaxies harbour more often dust ellipses (see Table 6). Thus more powerful radio sources occur preferentially in galaxies with regular (perhaps more "settled"?) dust.

Lanes and ellipses span the same range in size. There is no clear size difference for these dust morphologies in radio and non-radio galaxies. The compact and/or faint features which could be either lanes or ellipses form the smallest structures at a given distance. The irregular dust distributions tend to be the largest dust features. Larger-scale dust distributions tend to have a more irregular morphology.

The middle panel of Fig. 3 reveals no systematic difference between the host magnitude of each dust class for either the UGC FR-I or the UGC non-FR-I sample. Perhaps galaxies brighter than $M_{B} \sim-21.5$ do not contain dust with sizes below $\sim 200 \mathrm{pc}$, but this result is not statistically significant.

The bottom panel of Fig. 3 shows a correlation between dust orientation and morphology. The regular ellipses align within $\sim 10^{\circ}$ with the galaxy major axis, but the filamentary dust lanes do not. This result holds for both radio and non-radio galaxies and is also seen in the sample of lower-luminosity early-type galaxies of Tran et al. (2001). A corollary of these results is that the morphological classification based on appearance cannot be due (only) to viewing angle, but reflects (at least partly) intrinsic differences between dust lanes and ellipses.

The dust lanes in radio galaxies have $\triangle \mathrm{PA}_{\mathrm{DG}}$ values over the complete range, i.e., $\Delta \mathrm{PA}_{\mathrm{DG}} \sim\left[0^{\circ}, 90^{\circ}\right]$. In contrast, dust lanes in non-radio galaxies are within $\sim 25^{\circ}$ from either the minor- or major axis. It is not clear if this absence of large misalignment angles is simply due to the limited size of the sample. A similar absence was also seen by Tran et al. (2001) from WFPC2 imaging, but not by van Dokkum \& Franx (1995) using WFPC1 imaging. At the same time (and regardless of morphology) smaller sized ( $\lesssim 1 \mathrm{kpc}$ ) dust structures tend to align within $30^{\circ}$ of the galaxy major axis. Interestingly, only kpc-scale dust lanes, and the 120 pc dust lane in NGC 2768, are roughly aligned with the galaxy minor axis. NGC 2768 has large-scale irregular dust with an extent of $\sim 1.7 \mathrm{kpc}$ roughly along the minor axis of the galaxy, i.e., parallel to its small dust lane. This irregular dust is associated with an extended disk of emission-line gas which rotates around the major axis of the galaxy, i.e., orthogonally to the main motion of the stars (McDermid et al. 2004; Sarzi et al. 2005). The similarity between radio and non-radio galaxies in their relative orientations of dust lanes and ellipses, suggests, if taken at face value, that their orientations are not influenced by the presence of a radio jet.

\section{Relative position angles of dust, jet and host in radio galaxies}

We now focus on the position angle of dust lanes and ellipses relative to (i) the radio jet axis; and (ii) the galaxy major axis for the FR sample (see Table 1).

The left panel of Fig. 4 is similar to the bottom panel of Fig. 3, but now for the FR sample. It confirms the trends between the PA difference between dust and galaxy major axis $\triangle P A_{D G}$ and dust size of the subset of UGC FR-I galaxies in Fig. 3. The segregation in the location of the data points as a function of apparent dust morphology is now less clearcut. Dust ellipses still tend to align with the galaxy major axis (i.e., $\triangle \mathrm{PA} \mathrm{DG}_{\mathrm{DG}}<25^{\circ}$ ), but the alignment is not as tight as for the UGC FR-I sample by itself. Furthermore, there is one notable exception at $\triangle \mathrm{PA} A_{\mathrm{DG}} \sim 90^{\circ}$ (3C 76.1, see Sect. 9 for further discussion). Again, most lanes have large misalignments with the galaxy major axis (i.e., $\Delta \mathrm{PA}_{\mathrm{DG}}>20^{\circ}$ ) and the largest lanes align roughly with the galaxy minor axis.

The right panel of Fig. 4 shows $\triangle \mathrm{PA}_{\mathrm{DG}}$ as a function of the PA difference between dust and jet axis $\Delta \mathrm{PA}_{\mathrm{DJ}}$. We note three special features. First, no radio jets are observed close to the dust longest axis, i.e., $\Delta \mathrm{PA}_{\mathrm{DJ}}<20^{\circ}$. Second, the data points are distributed roughly along a mirrored "L" shape. Dust structures which are roughly aligned with the galaxy major axis $\left(\triangle \mathrm{PA} A_{\mathrm{DG}}<20^{\circ}\right)$ have a wide distribution in relative angles with the radio jet $\left(\triangle \mathrm{PA} A_{\mathrm{DJ}} \sim\left[20^{\circ}-90^{\circ}\right]\right)$. In contrast, dust features which are misaligned from the galaxy major axis $\left(\triangle \mathrm{PA} \mathrm{DG}>20^{\circ}\right)$ have a narrow distribution in PA differences with the radio jets $\left(\triangle \mathrm{PA} \mathrm{DJ} \sim\left[60^{\circ}-90^{\circ}\right]\right)$. These dust structures, while misaligned with the galaxy, are in a very rough sense perpendicular to the radio jets. Third, dust ellipses and lanes have a different distribution of $\triangle \mathrm{PA} \mathrm{DJ}_{\mathrm{DJ}}$. Ellipses have a wide distribution, $\Delta \mathrm{PA}_{\mathrm{DJ}} \sim 20^{\circ}-90^{\circ}$. In contrast, lanes have a narrow distribution: all lanes except one have $\Delta \mathrm{PA} A_{\mathrm{DJ}} \gtrsim 65^{\circ}$. The $\Delta \mathrm{PA} \mathrm{DJ}_{\mathrm{D}}$ of the dust lane galaxy $3 \mathrm{C} 353$, for which no $\triangle \mathrm{PA} \mathrm{DG}_{\mathrm{DG}}$ is available (Table 1), is consistent with this.

The FR sample contains 14 galaxies with dust features classified as "ambiguous between ellipse and lane" (cf. Table 1). $\triangle \mathrm{PA}_{\mathrm{DG}}$ and $\triangle \mathrm{PA} \mathrm{DJ}_{\mathrm{D}}$ can be measured for eight of them, and are plotted in Fig. 4. Their distribution among the lanes and ellipses supports the idea that in some cases these dust features are ellipses and in some cases they are lanes.

What (selection) effect(s) could create the mirrored L shape in the right panel of Fig. 4? It is not unreasonable to assume that jets ploughing through a dusty medium would quickly destroy the dust. This could explain the absence of systems with $\Delta \mathrm{PA}_{\mathrm{DJ}}<20^{\circ}$. Furthermore, suppose that jets are roughly perpendicular to the dust plane intrinsically. If the dust structures are intrinsically circular and viewed close to edge-on, then we will observe $\epsilon \sim 1$ in combination with $\triangle \mathrm{PA}_{\mathrm{DJ}} \sim 90^{\circ}$. If such a circular disk is viewed close to face-on, we will observe $\epsilon \sim 0$ in combination with a wide range of relative position angles, i.e., $\Delta \mathrm{PA}_{\mathrm{DJ}} \sim\left[0^{\circ}, 90^{\circ}\right]$. The thin filamentary dust lanes might be interpreted as systems viewed relatively close to edge-on. 


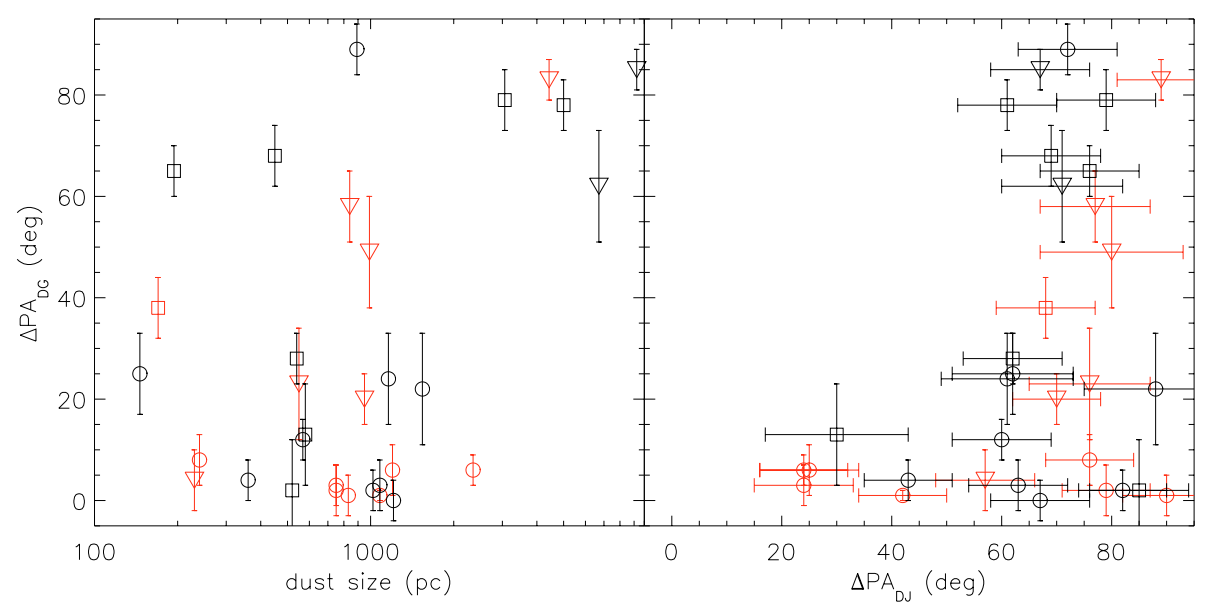

Fig. 4. Left: $\mathrm{PA}$ difference $\triangle \mathrm{PA} \mathrm{DG}_{\mathrm{D}}$ between dust axis and host galaxy major axis as a function of dust size for the FR sample. Galaxies with a dust ellipse, a dust lane or an "intermediate" morphology, i.e., in between lane and ellipse, are denoted by circles, triangles and squares, respectively. The subset of UGC FR-I galaxies have red symbols. The typical relative error on dust size is $10 \%$. The trends in the full FR sample are similar to those observed in the UGC FR-I and UGC non-FR-I sample as shown in Fig. 3. Right: $\triangle \mathrm{PA} \mathrm{DG}_{\mathrm{DG}}$ a function of position angle difference between dust and radio jet $\triangle \mathrm{PA}$ DJ

Their high values of $\triangle \mathrm{PA} \mathrm{DJ}_{\mathrm{DJ}} \gtrsim 60^{\circ}$ are then qualitatively consistent with radio jets being intrinsically approximately perpendicular to the dust plane.

\section{Are the dust ellipses intrinsically circular?}

Before analysing the intrinsic orientations of dust ellipses and radio jets, we test if the observed distribution of $\epsilon$ is consistent with circular or elliptical, thin or thick disks, assuming random viewing angles. This assumption is invalid if (i) the disk detection rate or classification depends on disk ellipticity; or (ii) the radio selection criteria result in a non-random distribution of jet viewing angles and there is a relation between disk and jet orientation. Scenario (i) is unlikely for the following reasons. We detect dust ellipses within almost the full range of possible ellipticities (i.e., 0.01 to 0.86 ). There is no correlation between observed ellipticity and distance. There is a dependence of classification on distance for dust structures classified as "intermediate" (i.e., between ellipse and lane). At distances $D<150 \mathrm{Mpc}, \sim 14 \%$ of dusty galaxies in the radio and non-radio galaxy samples are classified as intermediate. These do not have a clear bias for $\epsilon$ greater or smaller than 0.5 . However, nine of the eleven galaxies at $D>150 \mathrm{Mpc}$ have an ambiguous dust morphology classification of which six have $\epsilon \gtrsim 0.5$ (see Table 1). At these large distances it becomes impossible with the current imaging to distinguish unambiguously between high-ellipticity ellipses and lanes in general. Thus we limit the analysis of disk shapes to galaxies at $D<150 \mathrm{Mpc}$. Scenario (ii) does not seem to be relevant for the UGC FR-I sample as it is selected on total $1400 \mathrm{MHz}$ flux which is dominated by the extended and unbeamed radio-lobe emission. The radio cores from VLA $1490 \mathrm{MHz}$ measurements $\left(F W H M \sim 1.5^{\prime \prime}-3.75^{\prime \prime}\right)$ constitute always less than $22 \%$ of the total $1400 \mathrm{MHz}$ flux and typically $\sim 6 \%$ (Xu et al. 2000). Radio galaxies in the 3CR sample and B2 sample are selected using total $178 \mathrm{MHz}$ and $408 \mathrm{MHz}$ fluxes, respectively
(Bennett 1962; Colla et al. 1970). At these lower frequencies the radio emission is expected to be even more lobe-dominated.

We model dust ellipses as randomly-oriented, intrinsically triaxial bodies with an intermediate-to-long axis ratio $p$ and short-to-long axis ratio $q \leq p \leq 1$. Thus an infinitely thin circular disk has $p=1$ and $q=0$. We compare the observed distribution of $\epsilon$ to the expected distribution for given $p, q$ using the Kolmogorov-Smirnov (KS) test. Figure 5 shows the contour levels of equal KS probability in the plane of the axis ratios $p$ and $q$, for the sample of 18 radio galaxies with dust ellipses at $D<150 \mathrm{Mpc}$. The maximum $q$ allowed by the data is $q=0.14$, given that the maximum observed ellipticity is $\epsilon=0.86$. The contours are almost independent of $q$, suggesting that the data set is too small to put any further constraint on $q$. The KS probability is a function of $p$ and maximizes for close to oblate $(p \sim 0.74)$ disks. Similar results are obtained for the combined sample of 25 non-radio and radio galaxies at $D<150 \mathrm{Mpc}$ with dust ellipses. In the analysis of the three dimensional configuration of jets and dust disks, we will consider three representative models: a thin circular disk $(p=1, q=0)$, a thick circular disk ( $p=1, q=0.13$ ) and a thin elliptic disk $(p=0.75, q=0)$.

\section{Intrinsic orientation of dust ellipses and radio jets}

We now attempt to constrain the intrinsic orientation of the jet-axis relative to the dust systems classified as ellipses in the FR sample. Figure 6 illustrates the model parameters and coordinate system which we will use to describe the dust - jet system. The long, intermediate and short axes of the triaxial dust structure are chosen to lie along the $X, Y$ and $Z$-axes, respectively. The radio-jet axis makes an angle $\theta_{\mathrm{DJ}}$ with the $Z$-axis and an azimuthal angle $\phi_{\mathrm{DJ}}$ with the $X$-axis. The line of sight makes and angle $\theta_{\text {los }}$ with the $Z$-axis (i.e., the disk inclination angle) and an azimuthal angle $\phi_{\text {los }}$ with the $X$-axis. Thus, the disk-jet model has in general six parameters. 


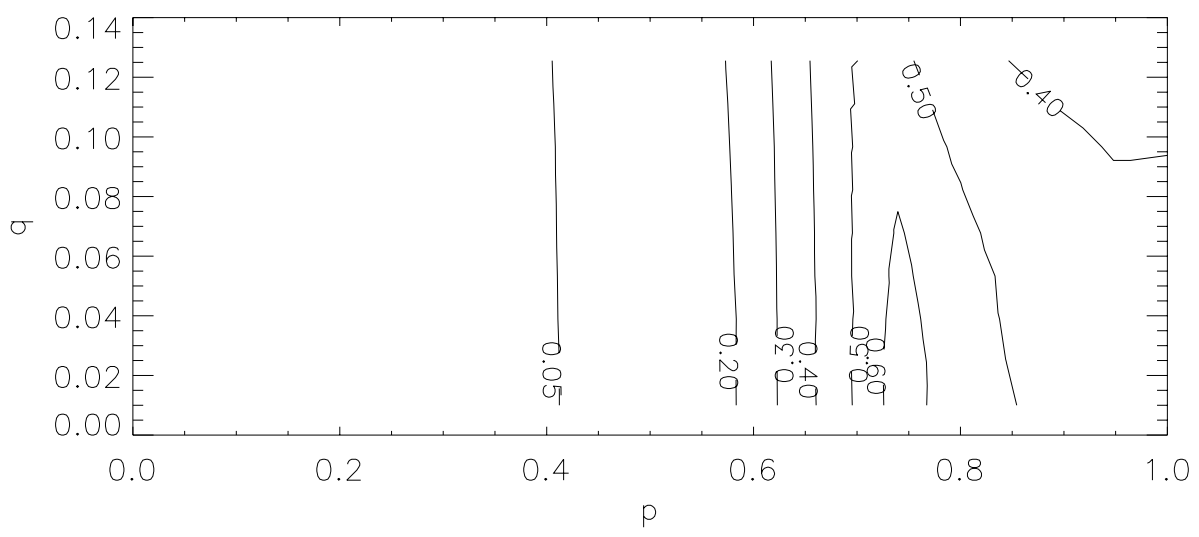

Fig. 5. Contours of constant probability using a Kolmogorov-Smirnov (KS) test for the triaxial properties of the dust ellipses. The hypothesis is that the ellipticities $\epsilon$ of the dust ellipses, observed in 18 F-I and FR-I/II galaxies at $D<150 \mathrm{Mpc}$, are caused by randomly-oriented triaxial bodies with an intermediate-to-long axis ratio $p$ and short-to-long axis ratio $q$. Each contour is labelled with its KS probability. The distribution of $\epsilon$ in the radio galaxies requires relatively thin $(q<0.14)$ disks and is most consistent with close to circular disks $(p>\sim 0.7)$. A similar result is obtained for the combined sample of 25 non-radio and radio galaxies with dust ellipses at $D<150 \mathrm{Mpc}$. See Sect. 6 for details.

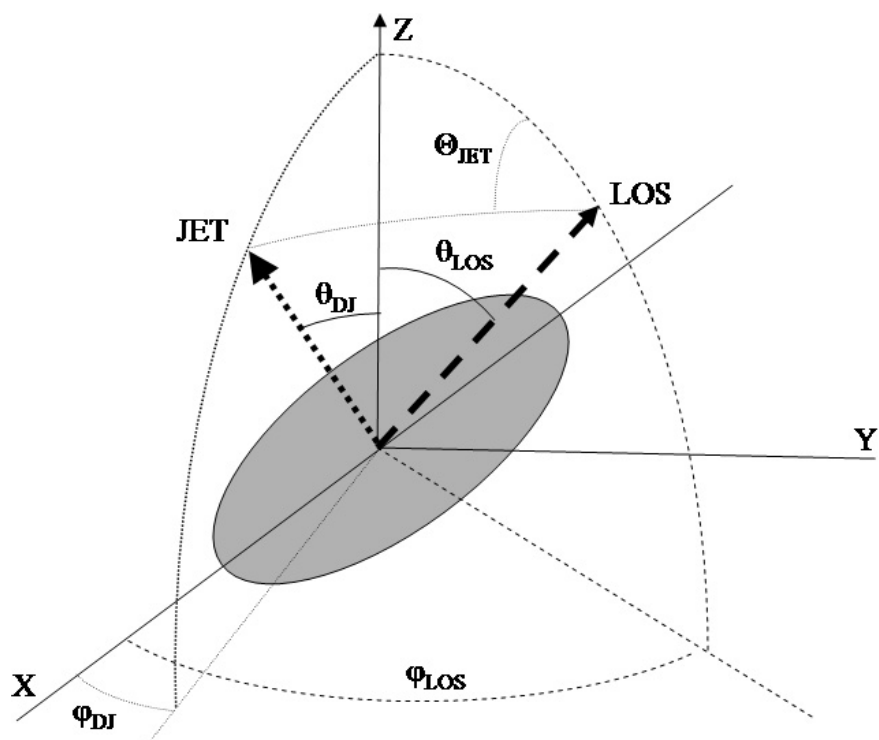

Fig. 6. The coordinate system used to describe the dust - jet system. The dust structure is assumed to be centred on the galaxy nucleus and to have a triaxial shape. The long, intermediate and short axis of the dust distribution lie along the $X, Y$ and $Z$-axis respectively. The jet axis is indicated by the dotted arrow and makes an angle $\theta_{\mathrm{DJ}}$ with the $Z$-axis and an azimuthal angle $\phi_{\mathrm{DJ}}$ with the $X$-axis. The line-of-sight direction, indicated by the dashed arrow, makes and angle $\theta_{\text {los }}$ with the $Z$-axis and an azimuthal angle $\phi_{\text {los }}$ with the $X$-axis. The spherical angle labelled $\Theta_{\text {jet }}$ indicates the angle between the jet and the short axis projected in the plane of the sky. See Sect. 7.1 for formulae relating these quantities.

\subsection{The relation between model parameters and observables}

The observations provide only two parameters of the dust-jet system as projected on the plane of the sky: the position angle difference between jet and disk $\Delta \mathrm{PA}_{\mathrm{DJ}}$ and the disk ellipticity $\epsilon$.
The ellipticity can be expressed in the model parameters as follows (Contopoulos 1956; Franx 1988):

$(1-\epsilon)^{2}=\frac{a-\sqrt{b}}{a+\sqrt{b}}$,

with

$\begin{aligned} a= & \left(1-q^{2}\right) \cos ^{2} \theta_{\mathrm{los}}+\left(1-p^{2}\right) \sin ^{2} \theta_{\mathrm{los}} \sin ^{2} \phi_{\mathrm{los}}+p^{2}+q^{2} \\ b= & {\left[\left(1-q^{2}\right) \cos ^{2} \theta_{\mathrm{los}}-\left(1-p^{2}\right) \sin ^{2} \theta_{\mathrm{los}} \sin ^{2} \phi_{\mathrm{los}}-p^{2}+q^{2}\right]^{2} } \\ & +4\left(1-p^{2}\right)\left(1-q^{2}\right) \sin ^{2} \theta_{\mathrm{los}} \cos ^{2} \theta_{\mathrm{los}} \sin ^{2} \phi_{\mathrm{los}} .\end{aligned}$

The position angle $\Theta_{\min }$ of the minor axis of the dust structure relative to the projection of the $Z$-axis (i.e., short axis) can be written as:

$\tan 2 \Theta_{\min }=\frac{2 T \sin \phi_{\mathrm{los}} \cos \phi_{\mathrm{los}} \cos \theta_{\mathrm{los}}}{\sin ^{2} \theta_{\mathrm{los}}-T\left(\cos ^{2} \phi_{\mathrm{los}}-\sin ^{2} \phi_{\mathrm{los}} \cos ^{2} \theta_{\mathrm{los}}\right)}$,

with triaxiality parameter $T=\left(1-p^{2}\right) /\left(1-q^{2}\right)$. The value of $\Theta_{\text {min }}$ indicates the position angle of the minor (rather than the major) axis if:

$\operatorname{sign}\left(\tan \Theta_{\min }\right)=\operatorname{sign}\left(\sin \phi_{\mathrm{los}} \cos \phi_{\mathrm{los}} \cos \theta_{\mathrm{los}}\right)$.

The position angle $\Theta_{\text {jet }}$ of the jet-axis relative to the position angle of the $Z$-axis can be expressed as (e.g., de Zeeuw \& Franx 1989):

$\tan \Theta_{\text {jet }}=\frac{\sin \Delta \phi \tan \theta_{\mathrm{DJ}}}{\sin \theta_{\mathrm{los}}-\cos \Delta \phi \tan \theta_{\mathrm{DJ}} \cos \theta_{\mathrm{los}}}$,

with $\Delta \phi=\phi_{\text {los }}-\phi_{\text {DJ }}$. The position angle difference $0^{\circ} \leq$ $\Delta \mathrm{PA}_{\mathrm{DJ}} \leq 90^{\circ}$ can then be expressed as:

$\cos \Delta \mathrm{PA}_{\mathrm{DJ}}=\sin \left(90^{\circ}-\Delta \mathrm{PA}_{\mathrm{DJ}}\right)=\left|\sin \left(\Theta_{\text {jet }}-\Theta_{\mathrm{min}}\right)\right|$.

Equation (3) shows that $\Theta_{\min }=0^{\circ}$ for a circular disk ( $p=1$ and $q=0)$, so that $\left|\sin \Theta_{\text {jet }}\right|$ corresponds to $\sin \left(90^{\circ}-\Delta \mathrm{PA}_{\mathrm{DJ}}\right)$. The expressions for $\epsilon$ and $\triangle \mathrm{PA} \mathrm{DJ}_{\mathrm{DJ}}$ then simplify to:

$\epsilon=1-\left|\cos \theta_{\mathrm{los}}\right|$, 


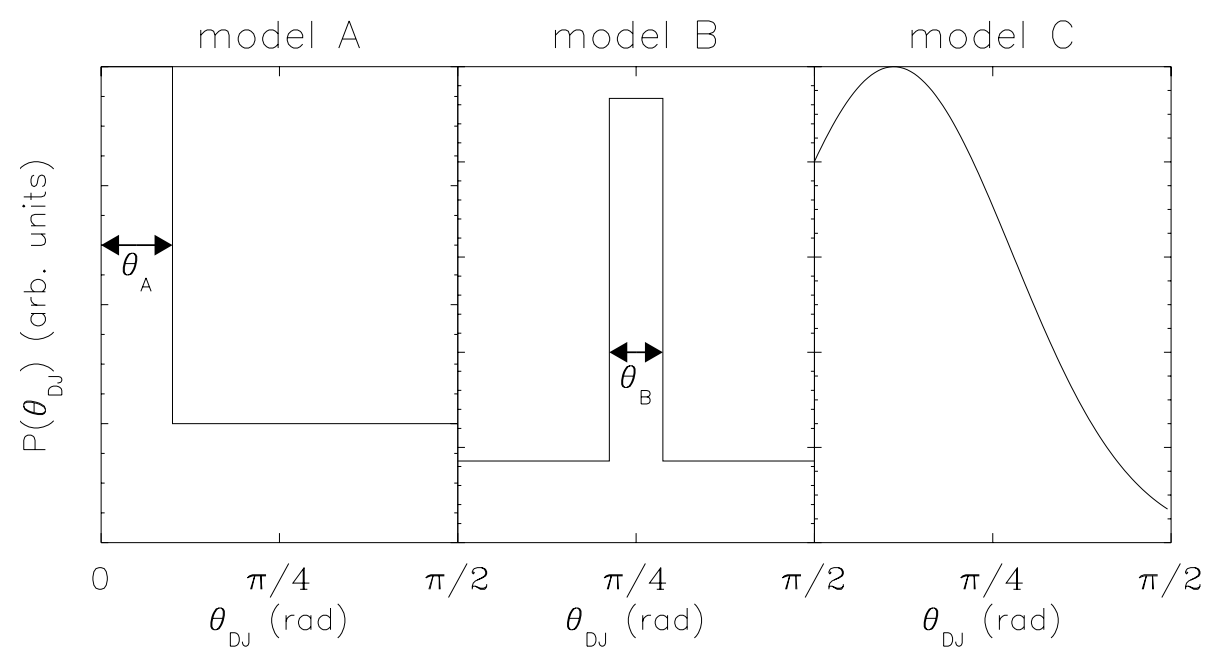

Fig. 7. The three probability distributions of the jet / dust disk misalignment angle $\theta_{\mathrm{DJ}}$ assumed to underlie the observations. Left: model A, the single-step function as described by Eq. (10). The width and height of the level at low $\theta_{\mathrm{DJ}}$ depend on the free parameter $\theta_{\mathrm{A}}$. The integrated probability underneath this level is $1-2 \theta_{\mathrm{A}} / \pi$. This model is used to test if the observations imply a peak in the $\theta_{\mathrm{DJ}}$ distribution at low or high $\theta_{\mathrm{DJ}}$. Middle: model B, the double-step function as described by Eq. (11). The width and height of the central level are set by $\theta_{\mathrm{B}}$ and is centred on $\theta_{\mathrm{DJ}}=\pi / 4$. The integrated probability under the central level is $1-2 \theta_{\mathrm{B}} / \pi$. Model B tests if the observations are consistent with a central peak or dip in the distribution of $\theta_{\mathrm{DJ}}$. Right: model C, a truncated Gaussian normalized to 1 (see Eq. (12)). Both the mean $0 \leq \mu \leq \pi / 2$ and dispersion $0 \leq \sigma \leq \pi$ are free parameters. This model tests for the presence of a peak anywhere in the range $0 \leq \theta_{\mathrm{DJ}} \leq \pi / 2$. See Sect. 7.3 for details.

and

$\tan \left(90^{\circ}-\Delta \mathrm{PA}_{\mathrm{DJ}}\right)=\left|\frac{\sin \Delta \phi \tan \theta_{\mathrm{DJ}}}{\sin \theta_{\mathrm{los}}-\cos \Delta \phi \tan \theta_{\mathrm{DJ}} \cos \theta_{\mathrm{los}}}\right|$

Thus $\Delta \mathrm{PA}_{\mathrm{DJ}}=90^{\circ}$ for any line of sight if $\theta_{\mathrm{DJ}}=0^{\circ}$.

\subsection{Lower limits on $\theta_{D J}$}

The angle between the line of sight and the radio jet is generally unknown. For an oblate dust structure (i.e., $p=1$ and $\Theta_{\min }=0^{\circ}$ ), the observed position angle difference with the disk merely confines the jet orientation to lie anywhere on the two "jet-circles" which are the two great circles defined by the line-of-sight vector and the angle $\Theta_{\text {jet }}$ (cf. Fig. 6 and Eq. (6); there are two jet-circles because $\Delta \mathrm{PA}_{\mathrm{DJ}}$ is an absolute value). The values of $\theta_{\mathrm{DJ}}$ and the difference in azimuthal angle $\Delta \phi$ fix the jet location in the jet-circles. We will refer to $\theta_{\mathrm{DJ}}$ as the "misalignment angle". To observe simultaneously a given position angle difference $\Delta \mathrm{PA}_{\mathrm{DJ}}<90^{\circ}$ and disk ellipticity $\epsilon$ requires a minimum misalignment angle $\theta_{\mathrm{DJ}}^{\mathrm{min}}$. Geometrically, this angle defines the latitude on a unit sphere which is tangent to the jet-circles. The jet axis intersects one of the two tangent points. The minimum misalignment angle can be expressed as (e.g., Schmitt et al. 2002)

$\sin \theta_{\mathrm{DJ}}^{\min }=\cos \Delta \mathrm{PA}_{\mathrm{DJ}} \sin \theta_{\mathrm{los}}$.

Since most radio galaxies have $\Delta \mathrm{PA}_{\mathrm{DJ}} \neq 90^{\circ}$ (Table 1 ), it follows that jets typically have $\theta_{\mathrm{DJ}}^{\min }>0^{\circ}$, irrespective of our line of sight towards the system. The $\theta_{\mathrm{DJ}}^{\min }$ for circular/oblate dust disks are listed in Table 1 . The maximum $\theta_{\mathrm{DJ}}^{\mathrm{min}}$ required by the FR sample is $54^{\circ}$ for $3 \mathrm{C} 449$ and four of the 16 disks for which $\triangle \mathrm{PA}_{\mathrm{DJ}}$ is available have $\theta_{\mathrm{DJ}}^{\min }>40^{\circ}$. If the disks are circular or oblate, significant misalignments occur between jet axis and disk normal.

\subsection{The distribution of $\theta_{D J}$ : Thin disks}

We have lower limits on $\theta_{\mathrm{DJ}}$ for individual circular disk-jet systems. The next step is to constrain the full distribution of $\theta_{\mathrm{DJ}}$. This constitutes an inversion problem: we have to recover the distribution functions of misalignment angles $\theta_{\mathrm{DJ}}$, azimuthal angles $\phi_{\mathrm{DJ}}$, and line-of-sight directions $\left(\theta_{\mathrm{los}}, \phi_{\mathrm{los}}\right)$ from the sample of observational pairs $\left(\Delta \mathrm{PA}_{\mathrm{DJ}}, \epsilon\right)$. We limit the analysis to the 18 dust ellipses at $D<150 \mathrm{Mpc}$ which are consistent with a spherically uniform random distribution of the line of sights (cf. Sect. 6). We assume that $\phi_{\mathrm{DJ}}$ has a uniform random distribution. The relative intrinsic orientation between jet and dust is characterized then by the misalignment angle distribution function $P_{\mathrm{DJ}}\left(\theta_{\mathrm{DJ}}\right)$. We interpret the distribution function as a probability density function. Given the small number of observations, we explore three parameterizations for $P_{\mathrm{DJ}}$ instead of attempting a parameter-free recovery. They are illustrated in Fig. 7. First, the observations are interpreted as being drawn from a step-function probability density distribution $P_{\mathrm{DJ}}\left(\theta_{\mathrm{DJ}}\right)$ (angles in radians):

$$
\begin{array}{ll}
P_{\mathrm{DJ}}\left(\theta_{\mathrm{DJ}}\right) \mathrm{d} \theta_{\mathrm{DJ}}=\frac{2}{\pi} \frac{\frac{\pi}{2}-\theta_{\mathrm{A}}}{\theta_{\mathrm{A}}} \mathrm{d} \theta_{\mathrm{DJ}}, & 0 \leq \theta_{\mathrm{DJ}} \leq \theta_{\mathrm{A}}, \\
P_{\mathrm{DJ}}\left(\theta_{\mathrm{DJ}}\right) \mathrm{d} \theta_{\mathrm{DJ}}=\frac{2}{\pi} \frac{\theta_{\mathrm{A}}}{\frac{\pi}{2}-\theta_{\mathrm{A}}} \mathrm{d} \theta_{\mathrm{DJ}}, & \text { otherwise. }
\end{array}
$$

The angle $\theta_{\mathrm{A}}$ is a free parameter. This step function, which we call model $\mathrm{A}$, tests the hypothesis that the distribution of $\theta_{\mathrm{DJ}}$ is peaked either at small or large misalignment angles or better agrees with a uniform distribution (i.e., $\theta_{\mathrm{A}}=\pi / 4$ ). Quantitatively, a fraction $1-2 \theta_{\mathrm{A}} / \pi$ of the jets have misalignment angles $\theta_{\mathrm{DJ}}<\theta_{\mathrm{A}}$, while the average $\theta_{\mathrm{DJ}}=\theta_{\mathrm{A}}$. Thus a $\theta_{\mathrm{A}}=\pi / 4$ corresponds to a uniform distribution in $\theta_{\mathrm{DJ}}$. Second, to test the hypothesis that the distribution of $\theta_{\mathrm{DJ}}$ does peak at intermediate misalignment angles (i.e., $\theta_{\mathrm{DJ}}=\pi / 4$ ) we 
analyse the data with the following two-step function, which we call model B:

$P_{\mathrm{DJ}}\left(\theta_{\mathrm{DJ}}\right) \mathrm{d} \theta_{\mathrm{DJ}}=\frac{2}{\pi} \frac{\frac{\pi}{2}-\theta_{\mathrm{B}}}{\theta_{\mathrm{B}}} \mathrm{d} \theta_{\mathrm{DJ}}, \quad \frac{\pi}{4}-\frac{\theta_{\mathrm{B}}}{2} \leq \theta_{\mathrm{DJ}} \leq \frac{\pi}{4}+\frac{\theta_{\mathrm{B}}}{2}$,

$P_{\mathrm{DJ}}\left(\theta_{\mathrm{DJ}}\right) \mathrm{d} \theta_{\mathrm{DJ}}=\frac{2}{\pi} \frac{\theta_{\mathrm{B}}}{\frac{\pi}{2}-\theta_{\mathrm{B}}} \mathrm{d} \theta_{\mathrm{DJ}}, \quad$ otherwise.

In this case a fraction of $1-2 \theta_{\mathrm{B}} / \pi$ jets have $\pi / 4-\theta_{\mathrm{B}} / 2<\theta_{\mathrm{DJ}}<$ $\pi / 4+\theta_{\mathrm{B}} / 2$ and the average $\theta_{\mathrm{DJ}}=\pi / 4$, regardless of $\theta_{\mathrm{B}}$. Thus $\theta_{\mathrm{B}}=\pi / 4$ corresponds to a uniform distribution in $\theta_{\mathrm{DJ}}$. Third, we explore a Gaussian distribution for $\theta_{\mathrm{DJ}}$ :

$P_{\mathrm{DJ}}\left(\theta_{\mathrm{DJ}}\right) \mathrm{d} \theta_{\mathrm{DJ}}=C \exp \left(-\frac{1}{2} \frac{\left(\theta_{\mathrm{DJ}}-\mu\right)^{2}}{\sigma^{2}}\right) \mathrm{d} \theta_{\mathrm{DJ}}, \quad 0 \leq \theta_{\mathrm{DJ}} \leq \frac{\pi}{2}$

The Gaussian is truncated to the physically allowed region $0 \leq$ $\theta_{\mathrm{DJ}} \leq \pi / 2$ and normalized to 1 using constant $C$. Both mean $\mu$ and dispersion $\sigma$ (of the full Gaussian) are left free to vary in the ranges $0 \leq \mu \leq \pi / 2$ and $0 \leq \sigma \leq \pi$. This model, which we call model $\mathrm{C}$, explores the presence of a peak not just at $\theta_{\mathrm{DJ}}=\pi / 4$ but anywhere and with a free width.

First, we estimate the $\theta_{\mathrm{A}}$ that best fits the observations using a Maximum Likelihood analysis. The goal is to maximize the likelihood $L$ of observing our data set of $\Delta \mathrm{PA}$ DJ and $\epsilon$ :

$L=\prod_{i=1}^{n_{\mathrm{obs}}} P\left(\Delta \mathrm{PA} \mathrm{DJ}, i_{i}, \epsilon_{i} \mid \theta_{\mathrm{A}}\right) \mathrm{d}\left(\Delta \mathrm{PA} A_{\mathrm{DJ}}\right) \mathrm{d} \epsilon$,

where the product is over the $n_{\text {obs }}$ observations. We approximate $P\left(\Delta \mathrm{PA}_{\mathrm{DJ}}, \epsilon \mid \theta_{\mathrm{A}}\right)$ using a Monte-Carlo simulation. We draw a large number of realizations of random line of sights $\left(\theta_{\mathrm{los}}, \phi_{\mathrm{los}}\right)$, random azimuthal jet angles $\phi_{\mathrm{jet}}$ and misalignment angles $\theta_{\mathrm{DJ}}$ from model A. Using Eqs. (7) and (8), these realizations are expressed in $\triangle \mathrm{PA}_{\mathrm{DJ}}$ and $\epsilon$. A two-dimensional histogram of the realizations with $n_{\text {bin }}^{2}$ equal-sized bins approximates $P\left(\Delta \mathrm{PA}_{\mathrm{DJ}, i}, \epsilon_{i} \mid \theta_{\mathrm{A}}\right)$ and hence the likelihood. The likelihood is maximized using an amoeba routine (Press et al. 1994) with $\theta_{\mathrm{A}}$ as the optimising parameter maximizing $L$. We choose the numerical simulation approach for two reasons. First, $P\left(\Delta \mathrm{PA}_{\mathrm{DJ}}, \epsilon\right)$ is not available in analytical form in general. Second, the Monte-Carlo simulation allows us to incorporate easily observational selection effects. For example, the three dust ellipses with $\epsilon<0.1$ are too roundish to determine $\triangle \mathrm{PA}$ DJ (see Sect. 3). We account for this by selecting a subset of $n_{\mathrm{mc}}$ pairs of $\left(\Delta \mathrm{PA} \mathrm{DJ}_{\mathrm{DJ}}, \epsilon\right)$ from the Monte-Carlo realizations which have $\epsilon>0.1$. We performed simulations using $n_{\mathrm{mc}}=10^{6}$ and $n_{\text {bin }}=10$. The numerical error arising from this choice is negligible compared to the uncertainties in $\theta_{\mathrm{A}}$ caused by the limited size and accuracy of the data set. Maximum Likelihood estimators are not guaranteed to be unbiased (e.g., Cowan 1998). We verified with simulations that the bias of the Maximum Likelihood estimator used here does not affect our results.

The Maximum Likelihood analysis infers $\theta_{\mathrm{A}}^{\mathrm{obs}}=43^{\circ}$ for the $n_{\text {obs }}=15$ observations with both $\Delta \mathrm{PA}_{\mathrm{DJ}}$ and $\epsilon>0.1$. We verify that the data are plausibly drawn from the best-fitting model by comparing the value of the Maximum Likelihood $L_{\mathrm{obs}}^{\max }$ of the observations to $L_{\mathrm{sim}}^{\max }$ of simulated data sets which were created using the best-fitting $\theta_{\mathrm{A}}^{\text {obs }}$. These mock samples contain the same number of galaxies as the observations. We find $L_{\mathrm{obs}}^{\max }>L_{\mathrm{sim}}^{\max }$ in $60 \%$ of the mock samples and hence the hypothesis is statistically well accepted.

We estimated the confidence levels on $\theta_{\mathrm{A}}^{\text {obs }}$ in a similar way. Figure 8 (top-left) shows the cumulative distribution function of $\theta_{\mathrm{A}}^{\text {sim }}$ inferred from 100 mock samples which are created in three ways:

1. In a Monte-Carlo fashion using the best-fitting $\theta_{\mathrm{A}}^{\text {obs }}$ in model A from the observations and the requirement that $\epsilon>0.1$.

2. "Dithering" the observations within the measurement errors.

3. By a bootstrap method, i.e., creating mock data sets of $\Delta \mathrm{PA}_{\mathrm{DJ}}, \epsilon$ by drawing $n_{\text {obs }}$ independent data pairs $\left(\Delta \mathrm{PA}_{\mathrm{DJ}}, \epsilon\right)$ from the $n_{\mathrm{obs}}$ observations.

The first method samples the uncertainty of the best fit due to the finite number of observations under the assumption that the underlying model is correct. The second method samples the uncertainty due to the random observational measurement errors. The third method samples a similar uncertainty as method 1 but with the observed data set itself used as an estimator of the underlying probability distribution.

All three methods infer median values for $\theta_{\mathrm{A}}^{\text {sim }} \sim 40^{\circ}-45^{\circ}$ in good agreement with the observations. The "dithering" method yields $1 \sigma, 2 \sigma$ confidence intervals which are significantly smaller than those of the other two methods. This shows that the statistical spread in $\theta_{\mathrm{A}}$ due to the finite sample size is the dominant source of uncertainty for this model. The confidence intervals from the Monte-Carlo and bootstrapping method are more similar, although the Monte-Carlo method shows the largest "probability wings" at small and large misalignment angles. Therefore we take the result from the Monte-Carlo method as a conservative estimate of the confidence levels.

We conclude that the observed jet-disk relative position angles and disk inclinations are consistent with a spherically random distribution of misalignment angles. The $1 \sigma$ upper and lower confidence levels indicate that the average $\theta_{\mathrm{DJ}}$ lies in the range $38^{\circ}-56^{\circ}$.

\subsection{The distribution of $\theta_{D J}$ : Thick disks}

In Sect. 6 we showed that the dust ellipses are not only consistent with thin circular disks (i.e., axis ratios $p=1$ and $q=0$ ), but also with oblate (i.e., thickened) disks, with axis ratios $p=1$ and $q=0.13$. For such structures, Eq. (1) simplifies to

$\epsilon=1-\sqrt{\cos ^{2} \theta_{\mathrm{los}}+q^{2} \sin ^{2} \theta_{\mathrm{los}}}$.

Thus, in comparison to thin disks, the thick disks appear rounder at any inclination $\theta_{\mathrm{los}}$. As a result, the inferred inclinations for the disks in the radio galaxy sample increase under the assumption of thick disks. Equation (9) then implies that the minimum misalignment angle $\theta_{\mathrm{DJ}}^{\min }$ increases for each disk. We redid the Maximum Likelihood analysis assuming dusty 


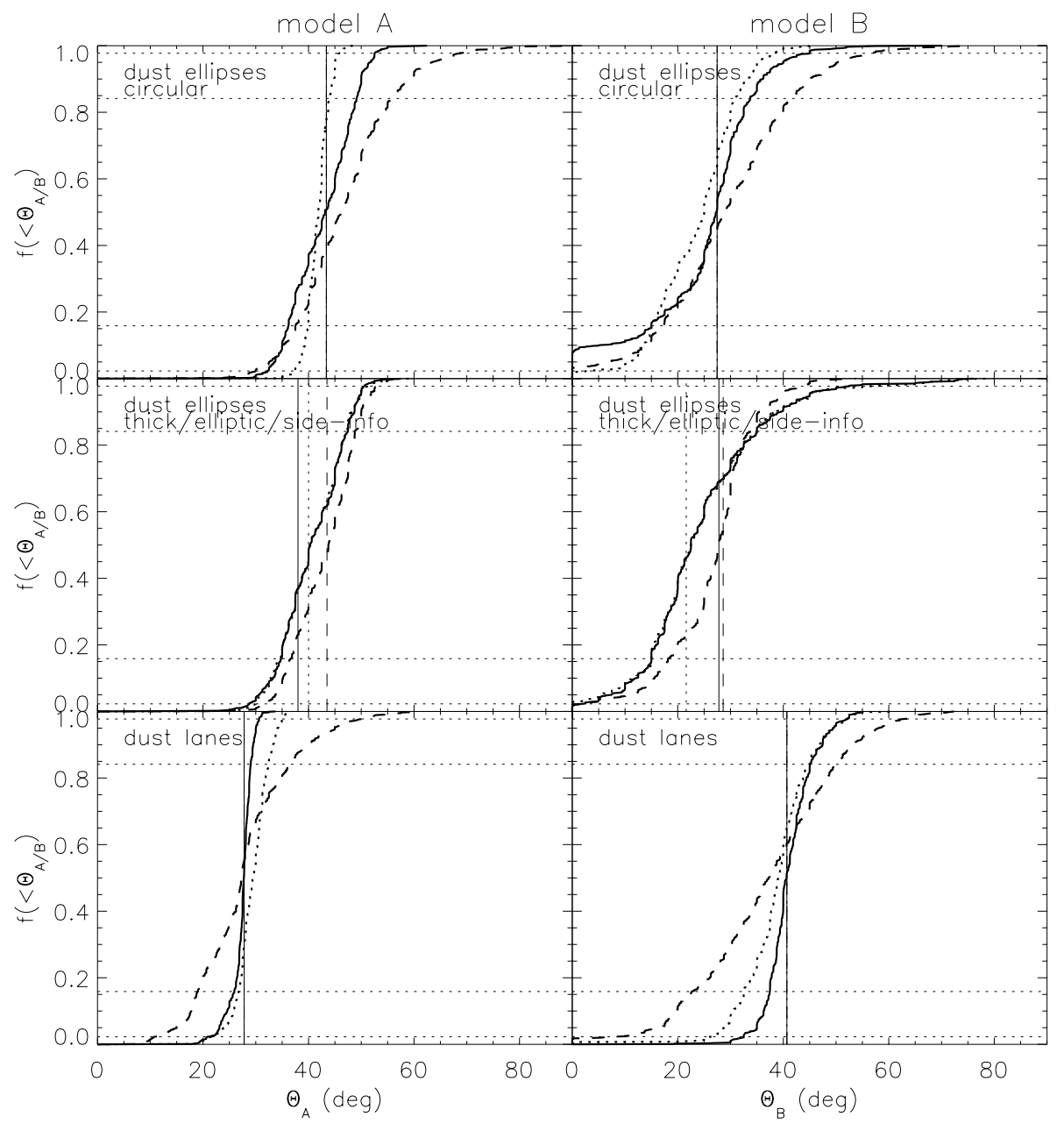

Fig. 8. Confidence levels on the best-fitted parameter $\theta_{\mathrm{A}}$ for model A (left column) and $\theta_{\mathrm{B}}$ for model B (right column) of the jet-dust misalignment angle, $\theta_{\mathrm{DJ}}$, distribution. Plotted are cumulative distributions of the best-fitted free parameter, $\theta_{\mathrm{A}}$ and $\theta_{\mathrm{B}}$, for various models of errors and dust morphology. The first four plots are models for the dust ellipses, the bottom two for the dust lanes. Top: thin circular disks are assumed ( $p=1$ and $q=0$ ). To estimate the error on $\theta_{\mathrm{A}}$ the bootstrap (solid), Monte-Carlo (dashed) and dither (dotted) method are used (see Sect. 7). The two-sided $68.27 \%$ and $95.45 \%$ confidence levels around the median are indicated by the horizontal dotted lines. The vertical lines indicate the best-fitted $\theta_{\mathrm{A}}$ and $\theta_{\mathrm{B}}$ for the observations. Middle: similar to the top model, but now for oblate "thick" disks ( $p=1, q=0.13$, dashed curve) and thin elliptic disks ( $p=0.75, q=0$, dotted curve) and for thin circular disks ( $p=1, q=0$, solid curve). The latter model uses additional information on the near side of jet and disk which is available for 10 of the 15 galaxies. All curves use the bootstrap method for error estimation. Bottom: similar to top diagrams, but now for eight lane galaxies. All models assume edge-on disks. The three methods of error estimation are indicated by the same line style as in the top diagrams. The main conclusion of the six diagrams is that dust lanes are most consistent with smaller misalignment angles than dust ellipses. The difference in misalignment angle is more clearly inferred by model $\mathrm{C}$ as shown in Fig. 9 . See Sects. 7 and 8 for a detailed discussion.

oblates with $q=0.13$. The analysis for thick disks yields as expected a slightly larger, but very similar $\theta_{\mathrm{A}}^{\text {obs }}=43.5^{\circ} \mathrm{com}-$ pared to thin disks. Also the confidence levels do not change significantly (see Fig. 8). Thus the inferred distribution of jet misalignment angles does not depend critically on the assumed thickness of the disks.

\subsection{The distribution of $\theta_{D J}$ : Elliptic disks}

We showed in Sect. 6 that the dust ellipses are also consistent with thin elliptic disks (i.e., $p=0.75$ and $q=0$ ) observed at random viewing angles. In contrast to circular disks, a $\triangle \mathrm{PA}_{\mathrm{DJ}}<90^{\circ}$ can be observed for certain viewing angles also for $\theta_{\mathrm{DJ}}=0^{\circ}$. However, only a fraction of the full range $0^{\circ} \leq \Delta \mathrm{PA}_{\mathrm{DJ}} \leq 90^{\circ}$ can be observed for certain $\epsilon$. Thus some dust ellipses still require a minimum misalignment angle $\theta_{\mathrm{DJ}}^{\min }>0^{\circ}$ in the case of an elliptic disk. We determined these angles numerically and they are listed in Table 1 next to those for the case of a circular disk. For three of the 16 dust ellipses $\theta_{\mathrm{DJ}}^{\min } \geq 40^{\circ}$ assuming $p=0.75$. Thus, significant misalignments do occur also if the dust ellipses are thin elliptic disks intrinsically.

We repeated the Maximum Likelihood analysis assuming that all dust ellipses are elliptic disks with $p=0.75$ and $q=0$. The result is plotted in the middle-left diagram in Fig. 8. As expected, the best-fitting average misalignment angle $\theta_{\mathrm{A}}^{\mathrm{obs}}=40^{\circ}$ is smaller than for thin circular disks. The change falls within the $1 \sigma$ confidence level for circular disks. Thus, the best-fit to 


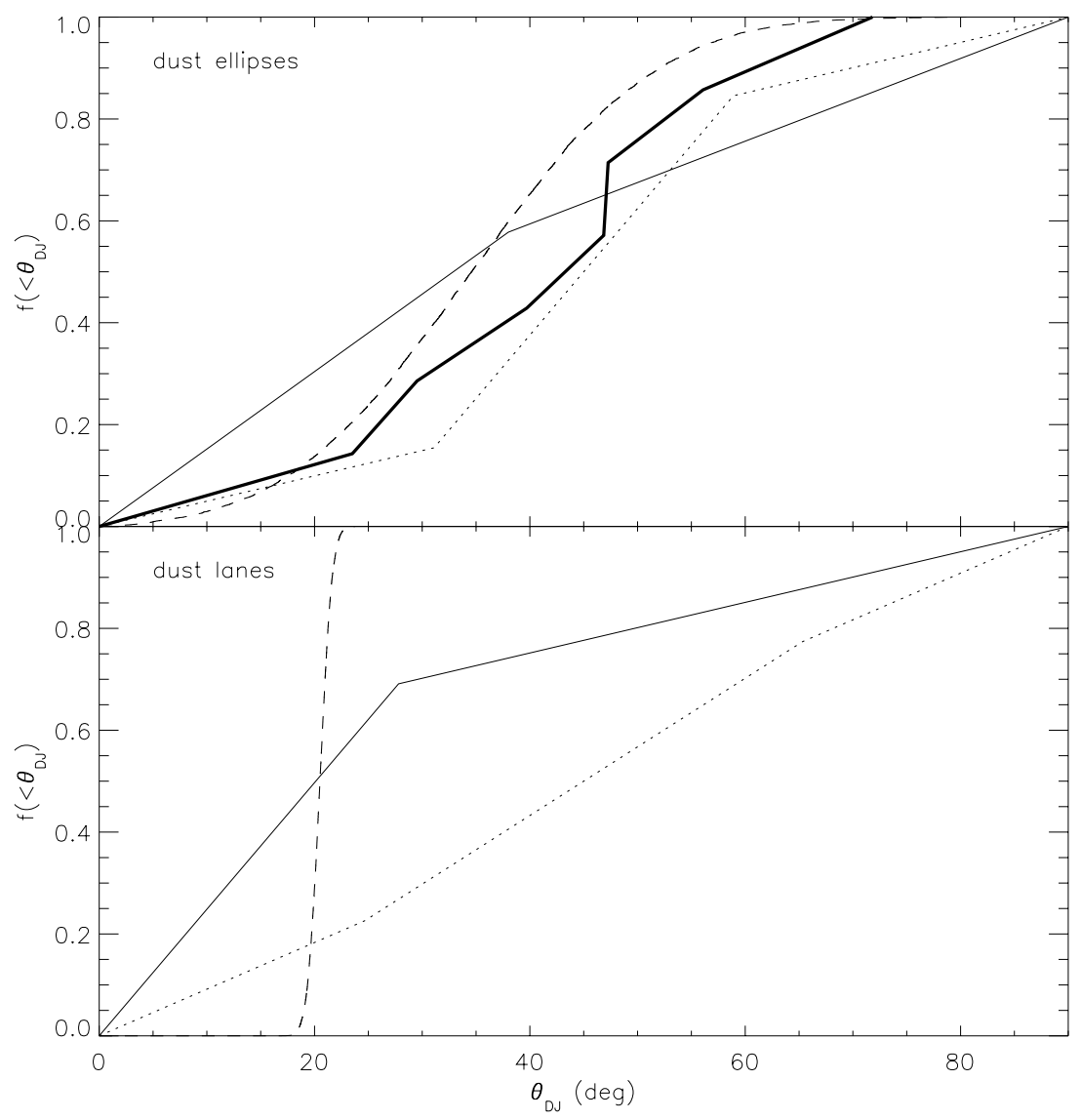

Fig. 9. Cumulative distributions of the jet-dust misalignment angle $\theta_{\mathrm{DJ}}$ for the radio galaxies with dust ellipses (top) and dust lanes (bottom). Top: the three thin curves indicate the cumulative distributions obtained from the Maximum Likelihood analyses of the observed distribution of dust ellipticities $\epsilon$ and dust-jet position angles differences $\Delta$ PA $A_{D J}$ from 15 FR-I and FR-I/II radio galaxies (see Sect. 7). The solid curve corresponds to model A (i.e., single-step function, cf. Eq. (10)), the dotted curve corresponds to model B (i.e., two-step function, cf. Eq. (11)) and the dashed curve for model C (i.e., the truncated Gaussian distribution, cf. Eq. (12)). The models assume thin circular dust disks and take into account the information on the near side of disk and jet, which is available for 10 radio galaxies (see Sect. 7.7). The thick solid curve indicates the cumulative distribution for the seven radio galaxies for which estimates of individual $\theta_{\mathrm{DJ}}$ are available (see Sect. 7.7). The shape of the latter curve does not depend much on the choice of $\theta_{\mathrm{DJ}}$ for $3 \mathrm{C} 296$ within its allowed range $30^{\circ} \leq \theta_{\mathrm{DJ}} \leq 73^{\circ}$ (we used the lower limit). Bottom: same as top panel, but now for the set of eight dust lanes. The lanes are assumed to be systems which are viewed edge-on (see Sect. 8). Correspondence between line style and model is similar to that in the top plot. The thick curve, present in the top plot, cannot be determined for dust lanes as $\epsilon$ and individual estimates of $\theta_{\mathrm{DJ}}$ are not available for lanes. The main conclusion is that jets of radio galaxies with dust lanes have on average smaller misalignment angles than those in radio galaxies with dust ellipses. See Sect. 8 for details.

the distribution of jet misalignment angles does not depend critically on the assumed ellipticity of the disks within the accepted range $p=[0.75,1]$.

\subsection{The peak in the distribution of misalignment angles}

The absence of peaks at small or large misalignment angles resulting from using model A cannot rule out a best-fit distribution of misalignment angles which peaks at an intermediate $\theta_{\mathrm{DJ}} \sim 45^{\circ}$. Thus it is worthwhile to explore model B which can test for such peaks. For circular disks, the analysis infers a bestfitting $\theta_{\mathrm{B}}^{\text {obs }}=27^{\circ}$, i.e., a broad peak corresponding to $\sim 70 \%$ of the jets having misalignment angles $\theta_{\mathrm{DJ}}=\left[32^{\circ}, 58^{\circ}\right]$. Figure 8 (top-right) shows the confidence levels using the three methods of parameter error estimation also used in Sect. 7.3. Although a broad peak is preferred, all methods indicate that neither a single $\theta_{\mathrm{DJ}}=45^{\circ}$ for all jets nor a distribution uniform in $\theta_{\mathrm{DJ}}$ (i.e., $\theta_{\mathrm{B}}=45^{\circ}$ ) can be ruled out at more than the $95 \%$ confidence level.

Figure 8 (middle-right) shows that the assumption of thick disks yields similar preferred $\theta_{\mathrm{B}}$, while assuming thin elliptic disks yields $\theta_{\mathrm{B}}=22^{\circ}$, i.e., a peak which is $5^{\circ}$ smaller in width. However the upper $95 \%$ confidence level on $\theta_{\mathrm{B}}$ increases, while small $\theta_{\mathrm{B}} \lesssim 10^{\circ}$ are ruled out at larger confidence compared to thin circular disks.

We explore model $\mathrm{C}$, the truncated Gaussian, to test for the presence of a peak in the $\theta_{\mathrm{DJ}}$ distribution outside $\theta_{\mathrm{DJ}}=45^{\circ}$. The best fitted truncated Gaussian from the Maximum Likelihood analysis indicates that at least half of the radio jets make an angle of $\sim 35^{\circ}$ or more with the symmetry axis of the dust disks (see Fig. 9). The confidence intervals on the free parameters $\mu$ and $\sigma$ are degenerate at large $\sigma$. The reason is the $\theta_{\mathrm{DJ}}$ distribution asymptotically approaches a uniform distribution 
regardless of the value of $\mu$. This precludes a direct interpretation of the confidence levels and hence we do not explore them.

In summary, the jet misalignment angle distribution in galaxies with dust ellipses is consistent with having a peak around $\theta_{\mathrm{DJ}} \sim 45^{\circ}$. The width of this peak is not well constrained. The limited data set cannot rule out a spherically random distribution of misalignment angles or a very narrow peak at more than $95 \%$ confidence. These conclusions do not depend critically on the assumed thickness or ellipticity. Regardless of the assumed model, typically at least half of the radio jets make an angle of $45^{\circ}$ or more with the symmetry axis of the dust disks.

\subsection{From axes to vectors}

In addition to the disk inclinations and dust-jet PA differences we can estimate the near side of dust disk and jet with respect to the observer in 11 of the radio galaxies (of which 10 are at $D<150 \mathrm{Mpc}$ ). This provides extra constraints on the distribution function of jet-disk misalignment angles. The flux and morphology asymmetries between the radio jets on both sides of the nucleus have been interpreted as being due to relativistic motion of the radio-emitting particles in intrinsically identical jets emerging on opposite sides from the nucleus (e.g., Laing et al. 1999). Thus the jet pointed nearest to the line of sight (i.e., the "main jet") appears brighter as the radio flux is either Doppler boosted or less Doppler dimmed in comparison to the jet on the other side of the nucleus. The column density of stellar light obscured by the dust disk along the line of sight is larger for the near side of the disk than for the far side. Thus one expects a difference in stellar surface brightness on both sides of the disk. Such an effect is indeed seen: in many cases, the major axis of the dust disk divides the disk into a more and a less obscured half. We identify the side with a lower stellar surface brightness with the near side of the disk. The surface brightness difference is not clearly present for disks with a low ellipticity. This is in qualitative agreement with the assumption that the disks are intrinsically close to circular in which case a lower ellipticity indicates a more nearly face-on disk for which the difference in the obscured amount of stellar light decreases. For 11 galaxies the observations reveal a clear radio flux asymmetry in the jets and a dust obscuration asymmetry which allows us to estimate the near side of disk and jet (cf. Table 1).

With the addition of near and far side information for jet and disk, the projections of the jet and dust axes in the plane of the sky become projections of a jet and dust vector. The main jet is projected against either the near side or far side of the dust disk (see Table 1). The absolute position angle difference between the two vectors can vary between $0^{\circ}$ and $180^{\circ}$ instead of the $0^{\circ}<\Delta \mathrm{PA}_{\mathrm{DJ}}<90^{\circ}$ for the dust and jet axis. We repeated the Maximum Likelihood analysis using this extra information for the ten galaxies at $D<150 \mathrm{Mpc}$. The $\theta_{\mathrm{A}}^{\text {obs }}$ inferred for model A decreases by $\$ 5^{\circ}$, indicating a slightly more peaked distribution of misalignment angles compared to the analysis without near side information (see Fig. 8, middle row). The inferred width of the peak at $\theta_{\mathrm{DJ}}=45^{\circ}$ for model B stays the same.
For seven of the 11 galaxies more detailed modelling of the jet asymmetries at radio frequencies has been published (see Table 1, for references). These studies report not only the near side of the jet but also an estimate of the viewing angle $\theta_{\mathrm{JL}}$ to the main jet (cf. Table 1). Under the assumption of circular disks, this viewing angle constrains the main jet to pass through either of the two points on each "jet-circle" at which the circle defined by $\theta_{\mathrm{JL}}$ intersects (cf. Sect. 7.2). These four points correspond to two different $\theta_{\mathrm{DJ}}$. For one point the main jet is projected against the near side of the dust disk while for the other point it is projected against the far side of the dust disk. Given our estimate of the near and far side of the dust disk, we can determine a unique $\theta_{\mathrm{DJ}}^{\text {radio }}$ which is listed in Table 1 . At least five of the seven misalignment angles are $\theta_{\mathrm{DJ}}^{\text {radio }} \geq 40^{\circ}$, confirming that such large misalignments occur frequently. Special support for this comes from the galaxies with $\theta_{\mathrm{DJ}}^{\text {radio }}$ which are much larger than the minimally required misalignment angle $\theta_{\mathrm{DJ}}^{\min }$. In fact, the distribution of $\theta_{\mathrm{DJ}}^{\text {radio }}$ agrees especially well with the distribution inferred from models $\mathrm{B}$ and $\mathrm{C}$ (see Fig. 9).

\section{Intrinsic orientation of dust lanes and radio jets}

We need to define a three-dimensional orientation for dust lanes to constrain the jet misalignment angles in radio galaxies with dust lanes. The analysis in Sect. 6 shows that the morphology of the dust ellipses is consistent with randomly oriented disks. The circumference of the dust lanes is too irregular to assign an ellipticity. It seems that the dust lanes are all viewed rather close to edge-on: the ratio of shortest to longest linear scale in the dust lanes is typically similar or smaller than the axis ratio of the most elongated dust ellipses in the sample.

To obtain an upper limit on the average misalignment, we will assume that all dust lanes are exactly edge-on systems. It follows from Eq. (9) that the minimally-required misalignment angle $\theta_{\mathrm{DJ}}^{\min }$ is maximal for $\theta_{\mathrm{los}}=90^{\circ}$, i.e., an edge-on disk. Thus we expect to first approximation that the Maximum Likelihood analysis will infer an upper limit to the median of the distribution of $\theta_{\mathrm{DJ}}$ if all lanes are assumed to be edge-on. We verified that this is indeed the case for the specific distribution of $\triangle \mathrm{PA}$ for the eight lanes at $D<150 \mathrm{Mpc}$.

The upper limit to the median misalignment angle for dust lane radio galaxies is smaller than the median angle for dust disk radio galaxies (cf. Fig. 9). This conclusion is reached by comparing models $\mathrm{A}, \mathrm{B}$ and $\mathrm{C}$. The inferred angle $\theta_{\mathrm{B}}=41^{\circ}$ suggests that the data are not consistent with a peak in the misalignment angle distribution at $\theta_{\mathrm{DJ}}=45^{\circ}$. On the contrary, the $\theta_{\mathrm{A}}=28^{\circ}$ and the typical misalignment angle $\theta_{\mathrm{DJ}} \sim 20^{\circ}$ from model $\mathrm{C}$ indicate that the observations are most consistent with such small misalignment angles. For model $\mathrm{A}$ the $\theta_{\mathrm{A}}$ inferred for the dust lanes falls outside the two-sided $\sim 95 \%$ confidence region inferred for dust ellipses (cf. Fig. 8). The exact confidence level depends on the assumed thickness, ellipticity of the dust disks and whether additional assumptions on near side of the jets and disks are included. Similarly, for model B, the inferred $\theta_{\mathrm{B}}$ falls outside the two-sided $\sim 68 \%$ confidence region for dust ellipses. 
The fact that dust lanes seem to be viewed close to edge-on prompts the question: where are the relatively face-on dust lanes? We explore several possibilities. It could be that close to face-on dust lanes are not classified by us as "lanes". At $D<150 \mathrm{Mpc}$ there are five galaxies with dust classified as intermediate between lane and disk. Three of these have axis ratios larger than 0.5 (i.e., roundish) and two have axis ratios less than 0.5 (i.e., flattened). Two of the three roundish galaxies have optical jets (3C 66B: Butcher et al. 1980 and 3C 78: Sparks et al. 1995), suggesting the jet is pointed close to the line of sight (e.g., Sparks et al. 2000). Thus at least these two galaxies are consistent with having dust structures which are close to face-on and having jets roughly perpendicular to them, i.e., face-on counterparts of dust-lane systems with perpendicular jets. Furthermore, the two galaxies with axis ratios $<0.5$ have $\Delta \mathrm{PA}_{\mathrm{DJ}}=68^{\circ}$ and $\Delta \mathrm{PA}_{\mathrm{DJ}}=76^{\circ}$, i.e., the large angles typical for the dust lane galaxies.

There are also five galaxies with dust classified as irregular. The dust in these galaxies typically shows several dusty filaments crossing the nucleus at several position angles. It is not implausible that these perturbed systems are the closer to faceon counterparts of perturbed dust lanes. The dust size distribution of the combined subset of galaxies with either intermediate dust or irregular dust is similar to that of dust lanes (see Fig. 4 and Table 1). In one galaxy with irregular dust (M 87) an optical jet is seen. In fact, three of the four galaxies at $D<150 \mathrm{Mpc}$ with optical jets in our sample are found in galaxies with dust classified as intermediate or irregular. The one other optical jet is in a dust disk galaxy (NGC 3862). This galaxy has a very round dust structure (Table 1). Thus one can speculate that this might also be a "face-on dust lane" galaxy where the jet is perpendicular to the disk (see also Sparks et al. 2000). It is interesting to note that two other round dust ellipses (NGC 541 and UGC 7115) do not have optical jets. Thus these could then be the dust disk galaxies for which we inferred that the jets are not perpendicular to the disks. In conclusion there is evidence that many of the ten galaxies which contain intermediate or irregular dust could be the closer to face-on counterparts of the eight rather edge-on dust lane galaxies.

\section{Discussion}

We repeat the three questions posed in the Introduction which form the focus of our analysis.

Are the properties of the fuel reservoirs different in radio galaxies and non-radio galaxies?

In terms of dust morphology the answer is yes: non-radio galaxies more often display highly irregular dust distributions. Perhaps this reflects the fact that the irregular fuel reservoirs are falling into the nucleus of the galaxy but are not feeding the black hole yet to cause nuclear activity and the formation of jets. In terms of reservoir orientation the answer is no. The orientations of dusty filaments and disks have similar distributions with respect to the optical isophotes for radio and non-radio galaxies.

Is the dust orientation governed by the gravitational potential?
Giant ellipticals are generally thought to be stationary or at most slowly rotating triaxial structures. Such galaxies have two equilibrium planes for dust disks, i.e., planes where stable non-intersecting orbits exist (e.g., Merritt \& de Zeeuw 1983). These planes are perpendicular to the short and long axis respectively. The angles between the minor axis and the short and long axis, respectively, depend on the line of sight and triaxiality $T=\left(1-p^{2}\right) /\left(1-q^{2}\right)$ of the host galaxy (e.g., Franx et al. 1991). If we assume that ellipses and lanes are both intrinsically circular structures, then their minor axis and hence $\Delta \mathrm{PA}_{\mathrm{DG}}$ constrain the rotation axis assuming that the dust is settled.

The top panel in Fig. 10 shows for the FR sample galaxies (at $D<150 \mathrm{Mpc}$ ) the observed cumulative distribution functions of $\triangle \mathrm{PA} \mathrm{DG}_{\mathrm{DG}}$ for dust ellipses and those predicted for randomly-oriented thin circular disks rotating around the short and/or long axis as a function of host triaxiality. The bottom panel shows the corresponding probabilities from a Kolmogorov-Smirnov test that the observed distribution is drawn from a model distribution with a specific stellar host triaxiality. In computing the KS probabilities we added typical errors to the model distributions. The errors have a skewed distribution because $0^{\circ}<\Delta \mathrm{PA} \mathrm{DG}<90^{\circ}$ by definition. Taking this into account significantly influences the probability distribution. The $\triangle \mathrm{PA} \mathrm{DG}_{\mathrm{D}}$ of ellipses are only consistent with disks rotating around the short axis. The preferred host triaxiality $T \sim 0.30$ is close to oblate, in agreement with early studies of giant ellipticals (e.g., Franx et al. 1991). In this scenario of settled disks, one would expect to first approximation (i) dust ellipticities which are always larger than stellar host ellipticities; and (ii) increasing host ellipticity for increasing disk ellipticity. This is exactly what is observed (Fig. 11).

Examining the properties of individual galaxies instead of general sample properties, we note two radio galaxies which could be exceptions to the above scenario: NGC 4261 and 3C 76.1. NGC 4261 is an unusual galaxy because the stellar mean motion is around the major axis (Davies \& Birkinshaw 1985). This could indicate that the galaxy is prolate. In that case the dust disk would lie in a plane which is not perpendicular to the long-axis. Such a plane is dynamically unstable and one would expect that the gas would have settled down in another configuration. Thus it is more likely that the galaxy is triaxial but close to prolate. In that case the orientation of the dust disk and mean rotation axis of the stars are consistent with the picture in which the dust disk lies in a plane perpendicular to the short axis of the galaxy, which is stable. The second stable plane in a triaxial galaxy, perpendicular to the long axis, is more unlikely given that the disk is elongated in the direction of the galaxy major axis. 3C 76.1 contains the only dust ellipse in the sample which is aligned with the minor axis of the galaxy instead of the major axis. Thus, this galaxy could be prolate with a settled dust disk in the stable plane perpendicular to the long-axis. Thus two out of 18 galaxies might be (nearly) prolate instead of (nearly) oblate. This fraction is consistent with the observed distribution of stellar rotation axes in large samples of ellipticals (Franx et al. 1991).

To constrain the host shape for dust lane galaxies we start out with assuming that also dust lanes are thin circular disks viewed at random viewing angles. Figure 10 shows that 

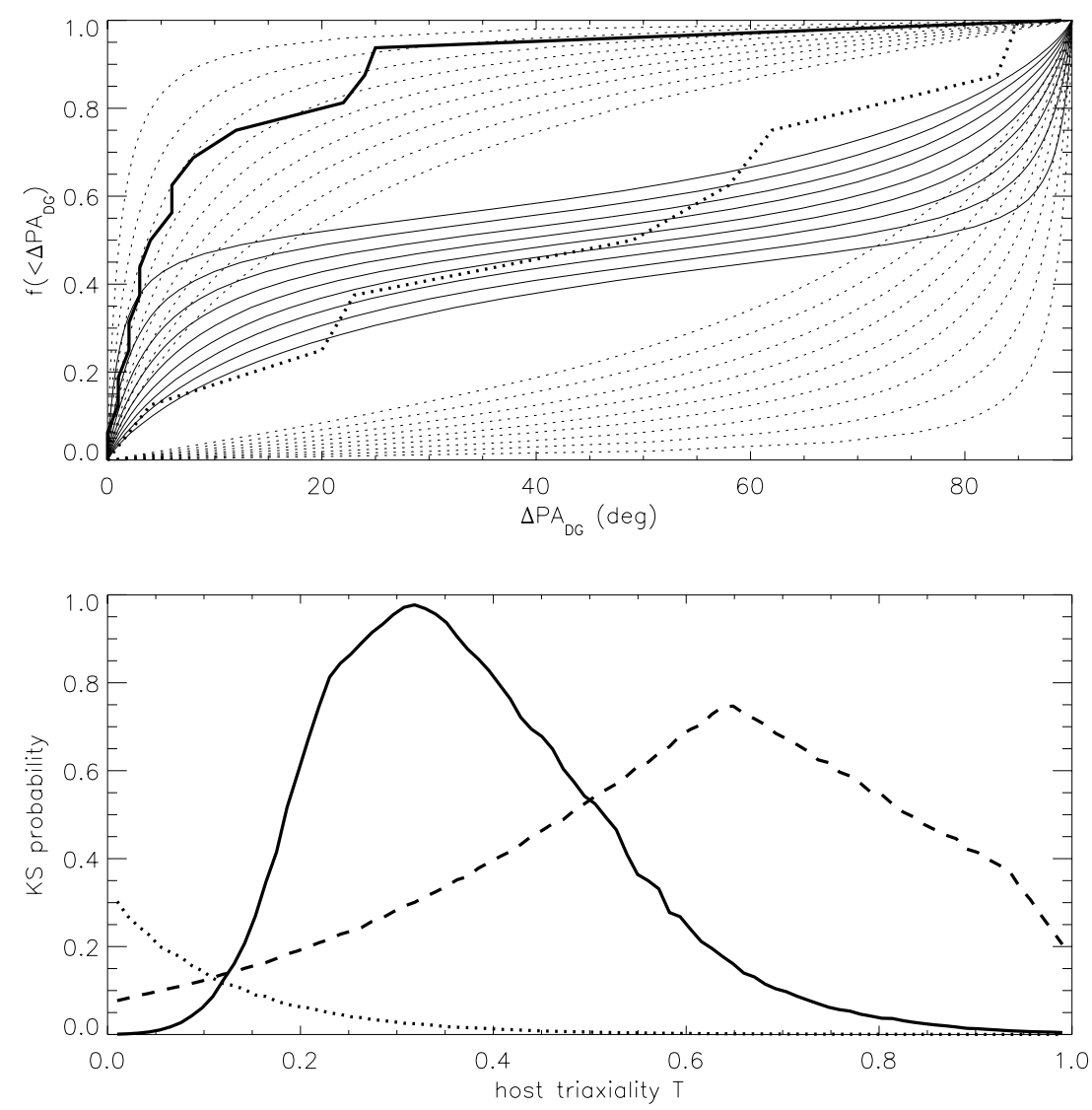

Fig. 10. Top: the cumulative distribution of $\triangle \mathrm{PA}_{\mathrm{DG}}$. The two thick lines show the observations for the FR sample galaxies at $D<150 \mathrm{Mpc}$ for dust ellipses (solid line) and dust lanes (dotted line). The thin dotted lines show the expected cumulative distribution of $\triangle \mathrm{PA} \mathrm{DG}_{\mathrm{D}}$ for circular disks rotating around the short axis (top series) and around the long axis (bottom series) assuming random viewing angles. The thin solid lines are the combination of these two distributions. They indicate the cumulative distribution for an equal number of disks rotating around the short and long axis. The nine curves in each series indicate the expectation for a stellar host triaxiality of 0.1 (top) increasing to 0.9 (bottom) in steps of 0.1. Bottom: the Kolmogorov-Smirnov (KS) probabilities that the observed $\Delta \mathrm{PA}_{\mathrm{DG}}$ distributions in the top plot are drawn from modelled distributions as a function of the stellar host triaxiality $T$. The computation assumes that the dust is a thin circular disk at random viewing angles and takes into account observational errors. The KS probability is significant at certain $T$ only for dust ellipses rotating around the short axis (solid line), lanes rotating around the long axis (dotted line) and lanes rotating in equal numbers around the short and long axis (dashed line). Figure 12 shows a similar analysis for dust lanes assuming viewing angles which are closer to edge-on. See Sect. 9 for further details.

the $\triangle \mathrm{PA} \mathrm{DG}_{\mathrm{D}}$ of dust lane galaxies is most consistent with equal numbers of dust lanes rotating around the short- and long axis in highly triaxial $(T \sim 0.65)$ galaxies. Such a high triaxiality appears inconsistent with other studies of the shapes of giant ellipticals (e.g., Franx et al. 1991). Furthermore, the similarity in large-scale axis ratios, optical luminosities and central stellar velocity dispersion suggests that dust lanes and dust ellipses live in similar hosts (Fig. 2). Figure 10 shows that the data cannot rule out at high confidence that lanes occupy systems with a typical triaxiality $T \sim 0.2-0.3$ but with lanes rotating around the long axis or around the short and long axis. However, as discussed in Sect. 8, the assumption of random viewing angles appears implausible as dust lanes are more likely to be viewed relatively close to edge-on. Thus, we repeat the KS analysis but now we constrain the viewing angles to lie within $30^{\circ}$ from edge-on. The result is shown in Fig. 12. The hypothesis that all lanes are dust structures settled in the equilibrium plane perpendicular to either the short or the long axis is ruled out with $>95 \%$ confidence. A model in which the dust lanes rotate in equal numbers around both axes cannot be ruled out at high confidence, but appears to be a poor fit to the observed $\triangle \mathrm{PA} \mathrm{DG}_{\mathrm{D}}$ as well.

The simultaneous differences between lanes and ellipses in both their $\triangle \mathrm{PA} \mathrm{DG}_{\mathrm{DG}}$ distribution and morphology support the alternative idea that the lanes are not simply settled, almost edge-on disks of dust. It is more likely that the dust lanes are perturbed disks. Models of a warped disk have been used to explain the filamentary dust morphology in NGC 4374 (M 84; Quillen \& Bower 1999) and both the morphology and kinematics of the interstellar matter in NGC 5128 (Quillen et al. 1992). For NGC 4374 and NGC 5128 the studies infer an angle between the line of sight and the symmetry axis of the warped disk of $68^{\circ}$ and $60^{\circ}$ respectively. In general, the perturbations and warps of the disks classified as "lanes" could be non-transient if they are caused by triaxiality of the gravitational potential or constantly perturbing forces related to the active galactic nuclei and jets. The perturbations would be transient if the dust lanes are in the process of settling down to become flat circular disks (Steiman-Cameron et al. 1992), at which point we would classify them as dust ellipses. There is 


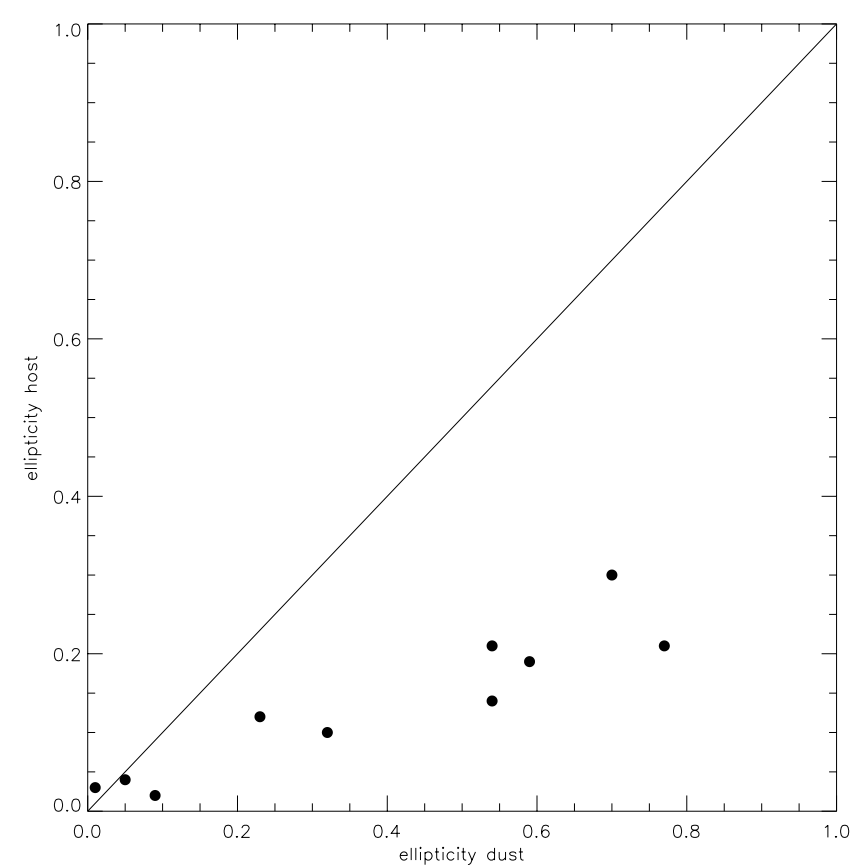

Fig. 11. The ellipticity of the stellar host as a function of dust ellipticity for the dust disks in the UGC FR-I sample. The host ellipticity is taken from HST imaging at a radius just outside the main dust distribution, using the results in Verdoes Kleijn et al. (1999). As expected for settled dust rotating around the short axis, the dust ellipses have ellipticities which are (i) systematically larger than host ellipticities; and (ii) increasing for increasing host ellipticity.

the following qualitative support for this. Some disks have in addition more irregular dust at larger scales (e.g., NGC 315, NGC 4335 and NGC 4261). In particular the case of NGC 4335 is interesting as there the larger scale dust is attached to the disk and has an arc-like morphology very reminiscent of a settling disk (e.g., Verdoes Kleijn et al. 2002). Some of the most regular lanes have small $\triangle \mathrm{PA}_{\mathrm{DG}}$ (e.g., NGC 5127 and NGC 5141). Furthermore, (some of) the five radio galaxies at $D<150 \mathrm{Mpc}$ with intermediate dust structures might be literally in between lane and ellipse. All have dust sizes $<550 \mathrm{pc}$ which is typical for the settled dust ellipses, but their dust orientation is not aligned with the galaxy major axis. For the nine cases at $D>150 \mathrm{Mpc}$ the classification might be better described as "ambiguous" due to large distance instead of intermediate (see Sect. 6). Lastly, the hosts of dust ellipses seem close to oblate in general. In oblate galaxies the dynamical settling times are largest for lanes perpendicular to the equatorial plane. At the same time, the settling process is most likely dissipational such that the dust structures become more compact as settling proceeds. Thus one expects larger lanes to have on average larger $\triangle \mathrm{PA} \mathrm{DG}_{\mathrm{D}}$. This is observed (see Fig. 3). Similarly, one expects large dust distributions to display closer alignment with the major axis at smaller radii because of the decreased dynamical time scale. NGC 4335 displays this behaviour, but it is not seen in general.

It is reasonable to assume that the current dust distribution was accreted by the galaxy before or around the time of the on-set of the AGN. The typical ages of FR I radio jets estimated from dynamical arguments is $\sim 10^{8} \mathrm{yr}$ and from radiative arguments $\sim 10^{7}$ yr (e.g., Eilek et al. 1997). Settling time-scales for the accreted gas are typically estimated to be a few orbital time-scales (e.g., Steiman-Cameron 1991). Orbital times are typically $\sim 2 \times 10^{6}(r / 100 \mathrm{pc})$ yr for giant ellipticals, where $r$ is the radius for a circular orbit. (This estimate assumes that the orbital circular velocities are on the order of the central velocity dispersions as measured in the central few $\operatorname{arcsec}^{2}$.) The lifetimes of the AGNs seem a bit long for the dust still to be settling down. However, the uncertainties in both time-scales are too large to make firmer statements.

Do jet/AGN related forces affect the dust orientation?

The dust/galaxy alignment dichotomy holds for giant elliptical galaxies in general, might they be radio or non-radio galaxies. A main result of our work is that for radio galaxies the dust/galaxy alignment dichotomy is connected to a jet misalignment dichotomy in three dimensions. Filamentary dust structures, i.e., the dust lanes, tend to be perpendicular to jets and to have an arbitrary orientation with respect to the galaxy major axis. Well-defined elliptical dust structures, i.e., the dust ellipses, have an arbitrary orientation with respect to the radio jets and tend to be aligned with the galaxy major axis. We explore qualitatively two scenarios to explain the dust/galaxy/jet orientation dichotomy.

Scenario I: the jets produce a torque in the hot X-ray gas. The torque is such that the X-ray gas tries to force the cooler dusty ISM in a plane perpendicular to the jet. Such a model was explored in detail for NGC 4374 by Quillen \& Bower (1999). The stellar mass potential produces a torque which tries to force the ISM in the stable settling planes of the galaxy potential. If the X-ray gas torque is stronger than the gravitational torque, the dust is forced in a plane perpendicular to the jet. This would produce the dust lanes galaxies. If the X-ray gas torque cannot overcome the gravitational torque the dust settles on non-intersecting orbits in the settling plane. This would produce the dust disk galaxies. It seems not implausible that an X-ray torque which is similar or stronger than the gravitational torque results in a perturbed disk with warps and filaments explaining the more irregular appearance of dust lanes compared to the dust ellipses (Quillen \& Bower 1999). This scenario does not explain why the dust/galaxy misalignment is also seen in galaxies without radio jets. However, it is interesting to note that none of the dust lanes in non-radio galaxies have $20^{\circ} \lesssim \Delta \mathrm{PA}_{\mathrm{DG}}<80^{\circ}$. In particular, small ( $\left.\lesssim \mathrm{kpc}\right)$ dust lanes are oriented close to the galaxy major axis and all large ( $\gtrsim \mathrm{kpc}$ ) dust lanes close to the minor axis. It might be that the small dust lanes in non-radio galaxies can be explained by dust structures settled in planes perpendicular to the short axis in a mildly triaxial host. The large-scale dust lanes in non-radio galaxies might be still settling down towards this plane. In contrast to the non-radio galaxies, quite a few radio galaxies have misalignments at $\triangle \mathrm{PA} \mathrm{DG}_{\mathrm{DG}} \sim 45^{\circ}$ and quite a few of those have small sizes. This difference could indicate that dust lanes in radio galaxies are prevented from settling down by an additional force.

Scenario II: dust is acquired externally (or from the outskirts of the galaxy) and the orientation of jet is aligned with the initial angular momentum of the dusty in-falling material. In this scenario the jet direction is determined during its 

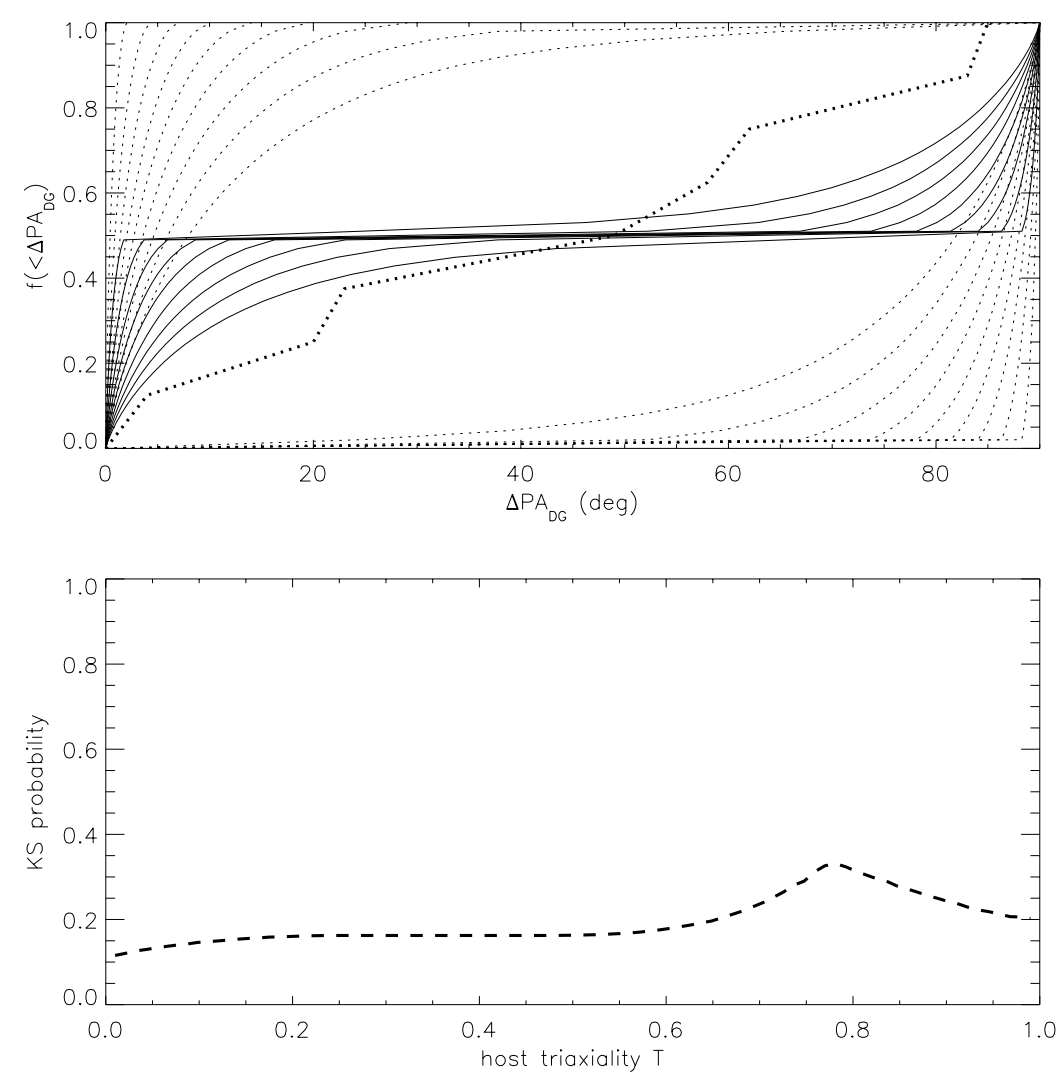

Fig. 12. Similar plots as Fig. 10, but now for a model in which the thin circular disks are viewed within $30^{\circ}$ from edge-on instead of fully random viewing angles. This model is more plausible for dust lanes. The Kolmogorov-Smirnov probabilities for lanes are only significant for lanes rotating around the short and long axis of the triaxial host. Nevertheless, this model does not provide a good fit to the data for any triaxiality $T$. In other words, it appears unlikely that the dust lanes are consistent with being settled structures in any of the two equilibrium planes in a triaxial host, but the data set is too small to rule out such a scenario completely.

formation by the angular momentum of in-falling material. A speculative example: it could be that an in-falling small galaxy brings in not only the dust and gas that we see, but also a central black hole. Initially the orientation of the dusty material then indicates the plane in which this black hole orbits the supermassive black hole of the giant elliptical. The angular momentum of the subsequent black hole binary or merger determines the orientation of the jets. Thus, initially the dust is expected to be perpendicular to the jet and to have a perturbed morphology because it has not settled yet. As time goes on the collisional dust and gas settles down in the galaxy potential thereby loosing its relation with the jet orientation. This scenario explains the dust/galaxy/jet dichotomy and the related differences in dust morphology. It also naturally explains why the dust/galaxy dichotomy is seen both in radio and non-radio galaxies. A weakness of the model is that the settling time-scales of the dust seem short compared to the age of the radio sources.

\section{Conclusions}

The main conclusions from the analysis of dust in 21 nearby FR-I radio galaxies and a comparison sample of 52 non-radio giant ellipticals are:

- The detection rate of dust is higher in radio galaxies $(\sim 90 \%)$ than in non-radio galaxies $(\sim 50 \%)$.
- The dust morphology can be classified as disk-like ellipses, filamentary lanes and irregular.

- Non-radio galaxies contain more often irregular dust distributions.

- The dust morphology, size, orientation relative to the stellar host of ellipses and lanes is similar in radio and non-radio galaxies.

- Filamentary dust, i.e., lanes, are generally misaligned with the galaxy major axis. Dust with a smooth elliptical appearance, i.e., dust ellipses, are roughly aligned with the galaxy major axis.

- The dust lanes and ellipses live in similar hosts in terms of stellar luminosity, axis ratios and velocity dispersions.

The main conclusions from the analysis of the relative orientation (in two and three dimensions) of dust, host galaxy and radio jet in a sample of 47 radio galaxies are:

- Dust ellipses are consistent with being relatively thin, circular or slightly elliptical disks rotating around the short axis of an oblate-triaxial gravitational potential with a triaxiality $T \sim 0.3$.

- The dust lanes are most likely warped or perturbed dust structures viewed rather close to edge-on. However, a scenario in which the dust lanes are settled structures in any of the two equilibrium planes of an oblate-triaxial galaxy cannot be ruled out completely. 
- The radio galaxies with dust with an apparent morphology classified as either irregular or intermediate between ellipse and lane most likely represent the close to face-on counterparts of the edge-on lanes.

- The radio jets do not have a particular orientation with respect to the dust disks. The mean angle between the disk normal and jet (the "misalignment angle") is inferred to be $\sim 45^{\circ}$.

- The radio jets tend to be perpendicular to the dust lanes, both in two and three dimensions. The upper limit to the mean misalignment angle is $\sim 20^{\circ}-30^{\circ}$.

- The above two results on jet orientations do not depend on the allowed range of ellipticity and thickness of the dust structures.

- The use of independent information on the near side of jets and dust disks strengthen the above results on jet/dust orientation.

There are two scenarios to explain the the dust/galaxy/jet orientation dichotomy for radio galaxies. Scenario I: the radio jets exert a torque on the nuclear dust in active galaxies which in some cases overcomes the gravitational torque causing the nuclear dust in a plane perpendicular to the jets. Scenario II: the angular momentum vector of unsettled nuclear dust is initially aligned with the radio jet in active galaxies but this alignment is lost as dust settles in an equilibrium plane of the galaxy.

To distinguish between these scenarios we deem the following steps relevant. First, larger samples of dusty galaxies, both active and inactive are required to confirm the dichotomy in jet/dust/galaxy orientation and its details. The current confidence levels on the existence of the dichotomy are $\$ 95 \%$. Second, more detailed analysis of host galaxy properties, such as gravitational potential and $\mathrm{X}$-ray gas pressure gradients, are required to obtain better estimates of the settling and life time of the dusty ISM. Third, combining such an analysis with detailed studies of the dusty and gaseous ISM, such as metallicity and two-dimensional kinematics, one might hope to constrain the origin of the dusty fuel reservoirs: stellar mass loss, cooling flows, or accretion of satellites.

Acknowledgements. Support for this work was provided by NASA through grant number \#GO-06673.01-95A from the Space Telescope Science Institute, which is operated by AURA, Inc., under NASA contract NAS5-26555. The authors thank Stefi Baum and Henrique Schmitt for discussions that helped to improve the manuscript, and Anne-Marie Weijmans for help in testing the software used in Sects. 7 and 8 . The authors are grateful to the referee for his/her comments. They helped to improve significantly the presentation of the results.

\section{References}

Balcells, M., Morganti, R., Oosterloo, T., Pérez-Fournon, J. I., \& González-Serrano, J. I. 1995, A\&A, 302, 665

Battistini, P., Bonoli, F., Silvestro, S., et al. 1980, A\&A, 85, 101

Bennett, A. S. 1962, MmRAS, 68, 163

Birkinshaw, M., \& Davies, R. L. 1985, ApJ, 291, 32
Butcher, H. R., van Breugel, W., \& Miley, G. K. 1980, ApJ, 235, 749

Capetti, A., \& Celotti, A. 1999, MNRAS, 304, 434

Capetti, A., de Ruiter, H. R., Fanti, R., et al. 2000, A\&A, 362, 871

Chiaberge, M., Capetti, A., \& Celotti, A. 2002, A\&A, 394, 791

Colla, G., Fanti, C., Ficarra, A., et al. 1970, A\&AS, 1, 281

Condon, J. J., \& Broderick, J. J. 1988, AJ, 96, 30

Contopoulos, G. 1956, Z. Astroph., 39, 126

Cowan, G. 1998, Statistical Data Analysis (Oxford: Clarendon Press) De Juan, L., Colina, L., \& Golombek, D. 1996, A\&A, 305, 776

de Koff, S., Baum, S. A., Sparks, W. B., et al. 1996, ApJS, 107, 621

de Koff S., Best, P., Baum, S. A., et al. 2000, ApJS, 129, 33

de Ruiter, H. R., Parma, P., Capetti, A., Fanti, R., \& Morganti, R. 2002, A\&A, 396, 857

de Zeeuw, P. T., \& Franx, M. 1989, ApJ, 343, 617

Dufour, R. J., Harvel, C. A., Martins, D. M., et al. 1979, AJ, 84, 284

Eilek, J. A., Melrose, D. B., \& Walker, M. A. 1997, ApJ, 483, 282

Faber, S., Wegner, G., Burstein, D., et al. 1989, ApJS, 69, 763

Fanaroff, B. L., \& Riley, J. M. 1974, MNRAS, 167, 31P

Fanti, C., Fanti, R., de Ruiter, H. R., \& Parma, P. 1986, A\&AS, 65, 145

Feretti, L., Perley, R., Giovannini, G., \& Andernach, H. 1999, A\&A, 341,29

Franx, M. 1988, MNRAS, 231, 285

Franx, M., Illingworth, G. D., \& de Zeeuw, P. T. 1991, ApJ, 383, 112

Goudfrooij, P., Hansen, L., Jorgensen, H. E., \& Norgaard-Nielsen, H. U. 1994, A\&AS, 105, 341

Guthrie, B. 1980, Ap\&SS, 70, 211

Hardcastle, M. J., Alexander, P., Pooley, G. G., \& Riley, J. M. 1997, MNRAS, 288, L1

Hardcastle, M. J., \& Worrall, D. M. 2000, MNRAS, 314, 359

Ho, L. C., Filippenko, A. V., \& Sargent, W. L. W. 1997, ApJ, 487, 568

Israel, F. P. 1998, A\&AR, 8, 237

Kauffmann, G., Heckman, T. M., Tremonti, C., et al. 2003, MNRAS, 346,1055

Knapp, G. R., Guhathakurta, P., Kim, D., \& Jura, M. A. 1989, ApJS, 70,329

Kormendy, J., \& Gebhardt, K. 2001, in The 20th Texas Symposium on Relativistic Astrophysics, ed. H. Martel, \& J. C. Wheeler (New York: AIP), 363

Kotanyi, C. G., \& Ekers, R. D. 1979, A\&A, 73, L1

Krajnović, D., \& Jaffe, W. 2002, A\&A, 390, 423

Laing, R. A., Parma, P., de Ruiter, H. R., \& Fanti, R. 1999, MNRAS, 306, 513

Laing, R. A., Canvin, J. R., \& Bridle, A. H. 2004, X-ray and radio connections, ed. L. O. Sjouwerman, \& K. K. Dyer, in press [arXiv:astro-ph/0405020]

Leahy, J. P., \& Perley, R. A. 1991, AJ, 102, 537

Martel, A. R., Baum, S. A., Sparks, W. B., et al. 1999, ApJS, 122, 81

McDermid, R., Emsellem, E., Cappellari, M., et al. 2004, AN, 325, 100

Merritt, D., \& de Zeeuw, P. T. 1983, ApJ, 267, L19

Möllenhoff, C., Hummel, E., \& Bender, R. 1992, A\&A, 255, 35

Nilson, P. 1973, Uppsala General Catalogue of Galaxies (Uppsala Astronomical Observatory), Uppsala

Noel-Storr, J., Baum, S. A., Verdoes Kleijn, G., et al. 2003, ApJS, 148, 419

Press, W. H., Teukolsky, S. A., Vetterling, W. T., \& Flannery, B. P. 1992, Numerical Recipes (2nd ed.; Cambridge: Cambridge University Press)

Quillen, A. C., de Zeeuw, P. T., Phinney, E. S., \& Phillips, T. G. 1992, ApJ, 391, 121

Quillen, A. C., \& Bower, G. A. 1999, ApJ, 522, 718

Roberts, M. S., Hogg, D. E., Bregman, J. N., Forman, W. R., \& Jones, C. 1991, ApJS, 75, 751 
Sadler, E. M., \& Gerhard, O. E. 1985, MNRAS, 214, 177

Sansom, A. E., Danziger, I. J., Ekers, R. D., et al. 1987, MNRAS, 229, 15

Sarzi, et al. 2005, MNRAS, submitted

Schmitt, H. R., Pringle, J. E., Clarke, C. J., \& Kinney, A. L. 2002, ApJ, 575,150

Schreier, E. J., Burns, J. O., \& Feigelson, E. D. 1981, ApJ, 251, 523

Spark, W. B., Golombek, D., Baum, S. A., et al. 1995, ApJ, 450, L55

Sparks, W. B., Baum, S. A., Biretta, J., Macchetto, F. D., \& Martel, A. R. 2000, ApJ, 542, 667

Steiman-Cameron, T. 1991, Warped Disks and Inclined Rings around Galaxies, ed. S. Casertano, P. D. Sackett, \& F. H. Briggs, 131

Steiman-Cameron, T. Y., Kormendy, J., \& Durisen, R. H. 1992, AJ, 104,1339
Tomita, A., Aoki, K., Watanabe, M., Takata, T., \& Ichikawa, S. 2000, AJ, 120, 123

Tonry, J. L., Dressler, A., Blakeslee, J. P., et al. 2001, ApJ, 546, 681

Tran, H. D., Tsvetanov, Z., Ford, H. C., et al. 2001, AJ, 121, 2928

van der Marel, R. P., \& van den Bosch, F. C. 1998, AJ, 116, 2220

van Dokkum, P. G., \& Franx, M. 1995, AJ, 110, 2027

Verdoes Kleijn, G. A., Baum, S. A., de Zeeuw, P. T., \& O’Dea, C. P. 1999, AJ, 118, 2592

Verdoes Kleijn, G. A., Baum, S. A., de Zeeuw, P. T., \& O'Dea, C. P. 2002, AJ, 123, 1334

Veron-Cetty, M.-P., \& Veron, P. 1988, A\&A, 204, 28

Xu, C., Baum, S. A., O’Dea, C. P., Wrobel, J. M., \& Condon, J. J. 2000, AJ, 120, 2950 